\title{
Detecting Whitney disks for link maps in the four-sphere
}

\author{
Ash Lightfoot \\ Faculty of Mathematics, \\ National Research University Higher School of Economics, \\ Usacheva str. 6, Moscow 119048, Russia \\ alightfoot@hse.ru
}

Received 16 January 2017

Revised 1 August 2017

Accepted 2 August 2017

Published 26 September 2017

\begin{abstract}
It is an open problem whether Kirk's $\sigma$-invariant is the complete obstruction to a link map $f: S_{+}^{2} \cup S_{-}^{2} \rightarrow S^{4}$ being link homotopic to the trivial link. The link homotopy invariant associates to such a link map $f$ a pair $\sigma(f)=\left(\sigma_{+}(f), \sigma_{-}(f)\right)$, and we write $\sigma=\left(\sigma_{+}, \sigma_{-}\right)$. With the objective of constructing counterexamples, Li proposed a link homotopy invariant $\omega=\left(\omega_{+}, \omega_{-}\right)$such that $\omega_{ \pm}$is defined on the kernel of $\sigma_{ \pm}$and which also obstructs link null-homotopy. We show that, when defined, the invariant $\omega_{ \pm}$ is determined by $\sigma_{\mp}$, and is strictly weaker. In particular, this implies that if a link map $f$ has $\sigma(f)=(0,0)$, then after a link homotopy the self-intersections of $f\left(S_{+}^{2}\right)$ may be equipped with framed, immersed Whitney disks in $S^{4} \backslash f\left(S_{-}^{2}\right)$ whose interiors are disjoint from $f\left(S_{+}^{2}\right)$.
\end{abstract}

Keywords: Link homotopy invariant; immersion; 2-sphere; Whitney disk; self-intersection number.

Mathematics Subject Classification 2010: 57N35, 57Q45

\section{Introduction}

A link map is a (continuous) map

$$
f: S^{p_{1}} \cup S^{p_{2}} \cup \cdots \cup S^{p_{n}} \rightarrow S^{m}
$$

from a union of spheres into another sphere such that $f\left(S^{p_{i}}\right) \cap f\left(S^{p_{j}}\right)=\emptyset$ for $i \neq j$. Two link maps are said to be link homotopic if they are connected by a homotopy through link maps, and the set of link homotopy classes of link maps as above is denoted $L M_{p_{1}, p_{2}, \ldots, p_{n}}^{m}$. It is a familiar result that $L M_{1,1}^{3}$ is classified by the linking number, and in his foundational work Milnor [15] described invariants of $L M_{1,1, \ldots, 1}^{3}$ which classified $L M_{1,1,1}^{3}$. These invariants (the $\bar{\mu}$-invariants) were refined much later by Habegger and Lin [5] to achieve an algorithmic classification of $L M_{1,1, \ldots, 1}^{3}$. 


\section{A. Lightfoot}

Higher dimensional link homotopy began with a study of $L M_{p, q}^{m}$ when $p, q \leq$ $m-3$, first by Scott [18] and later by Massey and Rolfsen [14]. Both papers made particular use of a generalization of the linking number, defined as follows. Given a link map $f: S^{p} \cup S^{q} \rightarrow S^{m}$, choose a point $\infty \in S^{m} \backslash f\left(S^{p} \cup S^{q}\right)$ and identify $S^{m} \backslash \infty$ with $\mathbb{R}^{m}$. When $p, q<m$, the map

$$
S^{p} \times S^{q} \rightarrow S^{m-1}, \quad(x, y) \mapsto \frac{f(x)-f(y)}{\|f(x)-f(y)\|}
$$

is nullhomotopic on the subspace $S^{p} \vee S^{q}$ and so determines an element $\alpha(f) \in$ $\pi_{p+q}\left(S^{m-1}\right)$. When $m=p+q+1$, the link homotopy invariant $\alpha$ is the integervalued linking number. In a certain dimension range, $\alpha$ was shown in [14] to classify embedded link maps $S^{p} \cup S^{q} \rightarrow S^{m}$ up to link homotopy. Indeed, historically, link homotopy roughly separated into settling two problems.

(1) Decide when an embedded link map is link nullhomotopic.

(2) Decide when a link map is link homotopic to an embedding.

In a large metastable range, this approach culminated in a long exact sequence which reduced the problem of classifying $L M_{p, q}^{m}$ to standard homotopy theory questions (see [10]).

On the other hand, the failure of the Whitney move in dimension four presents unique difficulties, and studying link homotopy of 2 -spheres in the 4 -sphere requires different techniques. In this setting, the first problem listed above was solved by Bartels and Teichner [1, who showed that an embedded link $S^{2} \cup S^{2} \cup \cdots \cup S^{2} \rightarrow S^{4}$ is link nullhomotopic. The present paper is concerned with invariants on $L M_{2,2}^{4}$ which have been introduced to address the second problem. Using techniques first introduced by Wall [19], the invariants obstruct equipping a link map with Whitney disks, by which one performs the Whitney move to eliminate self-intersections (see, for example, [4. Sec. 1.4]).

The first progress was made by Fenn and Rolfsen [2], who showed that $\alpha$ defines a surjection $L M_{2,2}^{4} \rightarrow \mathbb{Z}_{2}$ and in doing so constructed the first example of a link map $S^{2} \cup S^{2} \rightarrow S^{4}$ which is not link nullhomotopic. Kirk [8] generalized this result, introducing an invariant $\sigma$ of $L M_{2,2}^{4}$ which further obstructs embedding and surjects onto an infinitely generated group.

To a link map $f: S_{+}^{2} \cup S_{-}^{2} \rightarrow S^{4}$, where we use signs to distinguish component 2-spheres, Kirk defined a pair of integer polynomials $\sigma(f)=\left(\sigma_{+}(f), \sigma_{-}(f)\right)$ such that each component is invariant under link homotopy of $f$, determines $\alpha(f)$ and vanishes if $f$ is link homotopic to a link map that embeds either component. It is an open problem whether $\sigma$ is the complete obstruction; that is, whether $\sigma(f)=$ $(0,0)$ implies that $f$ is link homotopic to an embedding. By [1, Theorem 5], this is equivalent to asking if $\sigma$ is injective on $L M_{2,2}^{4}$. Seeking to answer in the negative, $\mathrm{Li}$ [12] proposed an invariant $\omega(f)=\left(\omega_{+}(f), \omega_{-}(f)\right)$ to detect link maps in the kernel of $\sigma$. 
When $\sigma_{+}(f)=0$, after a link homotopy the restricted map $\left.f\right|_{S_{+}^{2}}: S_{+}^{2} \rightarrow$ $S^{4} \backslash f\left(S_{-}^{2}\right)$ may be equipped with a collection of (framed, immersed) Whitney disks. Now, the interiors of these Whitney disks may intersect $f\left(S_{+}^{2}\right)$, and the invariant $\omega_{+}(f) \in \mathbb{Z}_{2}$ obstructs embedding by performing a weighted count of these intersections. Similarly, $\omega_{-}(f)$ is defined when $\sigma_{-}(f)=0$. Precise definitions of these invariants will be given in Sec. 2

It was shown in [13] that $\omega$ is an invariant of link homotopy, but the example produced in [12] of a link map $f$ with $\sigma(f)=(0,0)$ and $\omega(f) \neq(0,0)$ was found to be in error by Pilz [16. The purpose of this paper is to prove that $\omega$ cannot detect such examples; indeed, we have the following formula.

Theorem 1.1. Let $f$ be a link map with $\sigma_{-}(f)=0$ and let $a_{1}, a_{2}, \ldots, a_{N}$ be integers so that $\sigma_{+}(f)=\sum_{n} a_{n}\left(s^{n}-1\right)$. Then

$$
\omega_{-}(f)=\sum a_{n} \bmod 2,
$$

where the sum is over all $n$ equal to 2 modulo 4.

Consequently, there are infinitely many distinct classes $f \in L M_{2,2}^{4}$ with $\sigma_{-}(f)=0, \omega_{-}(f)=0$ but $\sigma_{+}(f) \neq 0$ (see Proposition 3.12). In particular, the following corollary answers [12], Question 6.2].

Corollary 1.2. If a link map $f$ has $\sigma(f)=(0,0)$, then $\omega(f)=(0,0)$.

Geometrically, Corollary 1.2 has the following consequence. (See Remark 2.7.)

Corollary 1.3. Let $f$ be a link map such that $\sigma(f)=(0,0)$. Then, after a link homotopy, the self-intersections of $f\left(S_{+}^{2}\right)$ may be paired up with framed, immersed Whitney disks in $S^{4} \backslash f\left(S_{-}^{2}\right)$ whose interiors are disjoint from $f\left(S_{+}^{2}\right)$.

This paper is organized as follows. In Sec. 2 we first review Wall intersection theory in the four-dimensional setting. The geometric principles thus established under the link homotopy invariants $\sigma$ and $\omega$, which we subsequently define. In Sec. 3 we exploit that, up to link homotopy, one component of a link map is an "unknotted" immersion, so as to equip this component with a convenient collection of Whitney disks which enable us to relate the invariants $\sigma$ and $\omega$. A more detailed outline of the proof may be found at the beginning of that section.

\section{Preliminaries}

Let us first fix some notation. For an oriented path or loop $\alpha$, let $\bar{\alpha}$ denote its reverse path; if $\alpha$ is a based loop, let $[\alpha]$ denote its based homotopy class. Let $*$ denote composition of paths from left to right, and denote the interval $[0,1]$ by $I$. We use $\equiv$ to denote equivalence modulo 2 , which for elements in an integer group ring we interpret as equality up to reducing coefficients modulo 2.

In what follows, assume all manifolds are oriented and equipped with basepoints; specific orientations and basepoints will usually be suppressed. 


\section{A. Lightfoot}

\subsection{Intersection numbers in 4-manifolds}

The link homotopy invariants investigated in this paper are closely related to the algebraic intersection "numbers" $\lambda$ and $\mu$ introduced by Wall [19]. For a more thorough exposition of the latter invariants in the four-dimensional setting, see [4. Chap. 1], from which our definitions are based.

Suppose $A$ and $B$ are properly immersed, self-transverse 2-spheres or 2-disks in a connected 4-manifold $Y$. (By self-transverse, we mean that self-intersections arise precisely as transverse double points.) Suppose further that $A$ and $B$ are transverse and that each is equipped with a path (a whisker) connecting it to the basepoint of $Y$. For an intersection point $x \in A \cap B$, let $\lambda(A, B)[x] \in \pi_{1}(Y)$ denote the homotopy class of a loop that runs from the basepoint of $Y$ to $A$ along its whisker, then along $A$ (avoiding all double points) to $x$, then back to the basepoint of $Y$ along $B$ (avoiding all double points) and its whisker. Define $\operatorname{sign}_{A, B}[x]$ to be 1 or -1 depending on whether or not, respectively, the orientations of $A$ and $B$ induce the orientation of $Y$ at $x$. The Wall intersection number $\lambda(A, B)$ is defined by the sum

$$
\lambda(A, B)=\sum_{x \in A \cap B} \operatorname{sign}_{A, B}[x] \lambda(A, B)[x]
$$

in the group ring $\mathbb{Z}\left[\pi_{1}(Y)\right]$, and is invariant under homotopy rel boundary of $A$ or $B$ [4, Proposition 1.7], but depends on the choice of basepoint of $Y$ and the choices of whiskers and orientations.

Let $A \cdot B \in \mathbb{Z}$ denote the usual signed intersection number; that is, the image of $\lambda(A, B)$ mapped forward via $\pi_{1}(Y) \mapsto\{1\}$.

For an element $h=\sum_{i} n_{i} g_{i}$ in $\mathbb{Z}\left[\pi_{1}(Y)\right]\left(n_{i} \in \mathbb{Z}, g_{i} \in \pi_{1}(Y)\right)$, define $\bar{h} \in$ $\mathbb{Z}\left[\pi_{1}(Y)\right]$ by $\sum_{i} n_{i} \overline{g_{i}}$. From the definition, it is readily verified that $\lambda(B, A)=$ $\overline{\lambda(A, B)}$ and that the following observations, which we record for later reference, hold.

Proposition 2.1. If $x, y \in A \cap B$, then the product of $\pi_{1}(Y)$-elements $\lambda(A, B)$ $[x] \overline{(\lambda(A, B)[y])}$ is represented by a loop that runs from the basepoint to $A$ along its whisker, along $A$ to $x$, then along $B$ to $y$, and back to the basepoint along $A$ and its whisker. Moreover, if $Y$ has abelian fundamental group, then this group element does not depend on the choice of whiskers and basepoint.

Proposition 2.2. If $D_{A} \subset A$ is an immersed 2-disk that is equipped with the same whisker and oriented consistently with $A$, then for each $x \in D_{A} \cap B$ we have $\lambda(A, B)[x]=\lambda\left(D_{A}, B\right)[x]$ and $\operatorname{sign}_{A, B}[x]=\operatorname{sign}_{D_{A}, B}[x]$.

The intersection numbers respect sums in the following sense. Suppose that $A$ and $B$ as above are 2-spheres, and suppose there is an embedded arc $\gamma$ from $A$ to $B$, with interior disjoint from both. Let $\iota_{A}$ be a path that runs along the whisker for $A$, then along $A$ to the initial point of $\gamma$, and let $\iota_{B}$ be a path that runs from the endpoint of $\gamma$, along $B$ and its whisker to the basepoint of $Y$. Form 
the connect sum $A \# B$ of $A$ and $B$ along $\gamma$ in such a way that the orientations of each piece agree with the result. Equipped with the same whisker as $B$, the 2-sphere $A \# B$ represents the element ${ }^{\mathrm{a}} A+g B$ in the $\mathbb{Z}\left[\pi_{1}(Y)\right]$-module $\pi_{2}(Y)$, where $g=\left[\iota_{A} * \gamma * \overline{\iota_{B}}\right] \in \pi_{1}(Y)$. If $C$ is an immersed 2-disk or 2-sphere in $Y$ transverse to $A$ and $B$, then $\lambda(A+g B, C)=\lambda(A, C)+g \lambda(B, C)$. The additive inverse $-A \in \pi_{2}(Y)$ is represented by reversing the orientation of $A$.

Allowing $A$ again to be a self-transverse 2-disk or 2-sphere, the Wall selfintersection number $\mu(A)$ is defined as follows. Let $f_{A}: D \rightarrow Y$ be a map with image $A$, where $D=D^{2}$ or $S^{2}$. Let $x$ be a double point of $A$, and let $x_{1}, x_{2}$ denote its two preimage points in $D$. If $U_{1}, U_{2}$ are disjoint neighborhoods of $x_{1}, x_{2}$ in int $D$, respectively, that do not contain any other double point preimages, then the embedded 2-disks $f_{A}\left(U_{1}\right)$ and $f_{A}\left(U_{2}\right)$ in $A$ are said to be two different branches (or sheets) intersecting at $x$. Let $\mu(A)[x] \in \pi_{1}(Y)$ denote the homotopy class of a loop that runs from the basepoint of $Y$ to $A$ along its whisker, then along $A$ (avoiding all other double points) to $x$ through one branch $f_{A}\left(U_{1}\right)$, then along the other branch $f_{A}\left(U_{2}\right)$ and back to the basepoint of $Y$ along $A$ (avoiding all other double points) and its whisker. (Such a loop is said to change branches at $x$.) Define $\operatorname{sign}_{A}[x]$ to be 1 or -1 depending on whether or not, respectively, the orientations of the two branches of $A$ intersecting at $x$ induce the orientation of $Y$ at $x$. In the group ring $\mathbb{Z}\left[\pi_{1}(Y)\right]$, let

$$
\mu(A)=\sum_{x} \operatorname{sign}_{A}[x] \mu(A)[x],
$$

where the sum is over all such self-intersection points. (Note that it may sometimes be more convenient to write $\mu\left(f_{A}\right)=\mu(A)$.) For a fixed whisker of $A$, changing the order of the branches in the above definition replaces $\mu(A)[x]$ by its $\pi_{1}(Y)$-inverse, so $\mu(A)$ is only well-defined in the quotient $Q(Y)$ of $\mathbb{Z}\left[\pi_{1}(Y)\right]$, viewed as an abelian group, by the subgroup $\left\{a-\bar{a}: a \in \pi_{1}(Y)\right\}$. The equivalence class of $\mu(A)$ in this quotient group is invariant under regular homotopy rel boundary of $A$. Note also that if the 4-manifold $Y$ has abelian fundamental group, then $\mu(A)$ does not depend on the choice of whisker.

Let $\operatorname{self}(A) \in \mathbb{Z}$ denote the signed self-intersection number of $A$. The reduced Wall self-intersection number $\hat{\mu}(A)$ may be defined by

$$
\hat{\mu}(A)=\mu(A)-\operatorname{self}(A) \in Q(Y) .
$$

It is an invariant of homotopy rel boundary [4, Proposition 1.7]. This observation derives from the fact that non-regular homotopy is distinguished by the occurrence of local "cusp" homotopies which may each change $\mu(A)$ by \pm 1 (see [4, Sec. 1.6]).

\footnotetext{
${ }^{\text {a Since }} A$ and $B$ are both whiskered, we permit ourselves to confuse them with their respective
} homotopy classes in $\pi_{2}(Y)$. 


\section{A. Lightfoot}

Finally, suppose further that the 4-manifold $Y$ is a codimension-0 submanifold of $S^{4}$ and that $A$ is a 2 -sphere. Then if $A^{\prime}$ is the image of a section of the normal bundle of $A$, we have

$$
\lambda\left(A, A^{\prime}\right) \equiv \mu(A)+\overline{\mu(A)},
$$

so in the reduced group ring $\mathbb{Z}_{2}\left[\pi_{1}(Y)\right]$ we may unambiguously set $\lambda(A, A)=$ $\lambda\left(A, A^{\prime}\right)$.

\subsection{The link homotopy invariants}

We now recall the definitions of the link homotopy invariants $\sigma$ of Kirk 8 and $\omega$ of $\mathrm{Li}[12$.

Let $f: S_{+}^{2} \cup S_{-}^{2} \rightarrow S^{4}$ be a link map. After a link homotopy (in the form of a perturbation) of $f$, we may assume the restriction $f_{ \pm}=\left.f\right|_{S_{ \pm}^{2}}: S_{ \pm}^{2} \rightarrow S^{4} \backslash f\left(S_{\mp}^{2}\right)$ to each component is a self-transverse immersion; let $X_{ \pm}=S^{4} \backslash f\left(S_{ \pm}^{2}\right)$. Choose a generator $s$ for $H_{1}\left(X_{-}\right) \cong \mathbb{Z}$, which we write multiplicatively. For each double point $p$ of $f\left(S_{+}^{2}\right)$, let $\alpha_{p}$ be a simple circle on $f\left(S_{+}^{2}\right)$ that changes branches at $p$ and does not pass through any other double points. We call $\alpha_{p}$ an accessory circle for $p$. Letting $n(p)=\operatorname{lk}\left(\alpha_{p}, f\left(S_{-}^{2}\right)\right)$, one defines

$$
\sigma_{+}(f)=\sum_{p} \operatorname{sign}(p)\left(s^{|n(p)|}-1\right)
$$

in the ring $\mathbb{Z}[s]$ of integer polynomials, where the sum is over all double points of $f\left(S_{+}^{2}\right)$, and to simplify notation we write $\operatorname{sign}(p)=\operatorname{sign}_{f_{+}\left(S^{2}\right)}[p]$.

Reversing the roles of $f_{+}$and $f_{-}$, we similarly define $\sigma_{-}(f)$ and write $\sigma(f)=$ $\left(\sigma_{+}(f), \sigma_{-}(f)\right) \in \mathbb{Z}[s] \oplus \mathbb{Z}[s]$. Kirk showed in $[8]$ that $\sigma$ is a link homotopy invariant, and in [9] that if $f$ is link homotopic to a link map for which one component is embedded, then $\sigma(f)=(0,0)$.

Let $\rho: \pi_{1}\left(X_{-}\right) \rightarrow H_{1}\left(X_{-}\right)=\mathbb{Z}\langle s\rangle$ denote the Hurewicz map composed with the above identification (necessarily given by $\rho(\alpha)=s^{\varepsilon \cdot \operatorname{lk}\left(\alpha, f\left(S_{-}^{2}\right)\right)}$, where $\varepsilon=1$ or $-1)$. Referring to the definition of $\mu$ in the preceding section as applied to the map $f_{+}: S_{+}^{2} \rightarrow X_{-}$, observe that $\rho$ carries $Q\left(X_{-}\right)$to the ring of integer polynomials $\mathbb{Z}[s]$ and Kirk's invariant $\sigma_{+}$is given by

$$
\sigma_{+}(f)=\rho\left(\hat{\mu}\left(f_{+}\right)\right) \in \mathbb{Z}[s] .
$$

As in [13], we say that $f$ is \pm -good if $\pi_{1}\left(X_{\mp}\right) \cong \mathbb{Z}$ and the restricted map $f_{ \pm}$is a self-transverse immersion with $\operatorname{self}\left(f_{ \pm}\right)=0$. We say that $f$ is $\operatorname{good}$ if it is both + - and --good. Equation (2.1) has the following consequence.

Proposition 2.3. If $f$ is $a \pm$-good link map, then $\sigma_{ \pm}(f)=\mu\left(f_{ \pm}\right)$.

The invariant $\sigma_{+}(f)$ obstructs, up to link homotopy, pairing up double points of $f\left(S_{+}^{2}\right)$ with Whitney disks in $X_{-}$, and we have a symmetric statement for $\sigma_{-}(f)$. 
While the essential purpose of Whitney disks is to embed (or separate) surfaces, our focus will be on their construction for the purposes of defining certain invariants. In the setting of link maps, the following standard result (phrased in the context of link maps) is the key geometric insight behind all the invariants we discuss in this paper and will find later application.

Lemma 2.4. Let $f$ be a link map such that $f_{-}$is a self-transverse immersion, and suppose $\left\{p^{+}, p^{-}\right\}$are a pair of oppositely-signed double points of $f\left(S_{-}^{2}\right)$. Let $U$ and $V$ each be an embedded 2-disk neighborhood of $\left\{p^{+}, p^{-}\right\}$on $f\left(S_{-}^{2}\right)$ such that $U$ and $V$ intersect precisely at these two points. On $f\left(S_{-}^{2}\right)$, let $\alpha^{+}, \alpha^{-}$be loops based at $p^{+}, p^{-}$(respectively) that leave along $U$ and return along $V$. Let $\gamma_{U}, \gamma_{V}$ be oriented paths in $U, V$ (respectively) that run from $p^{+}$to $p^{-}$. Then the oriented loop $\gamma_{U} \cup \overline{\gamma_{V}}$ satisfies

$$
\left|\operatorname{lk}\left(\gamma_{U} \cup \overline{\gamma_{V}}, f\left(S_{+}^{2}\right)\right)\right|=\left|\operatorname{lk}\left(\alpha^{+}, f\left(S_{+}^{2}\right)\right)-\operatorname{lk}\left(\alpha^{-}, f\left(S_{+}^{2}\right)\right)\right|
$$

Wishing to obtain a "secondary" obstruction, in [12] Li proposed the following $\left(\mathbb{Z}_{2} \oplus \mathbb{Z}_{2}\right)$-valued invariant to measure intersections between $f\left(S_{+}^{2}\right)$ and the interiors of its Whitney disks in $X_{-}$.

Suppose $f$ is a -good link map with $\sigma_{-}(f)=\mu\left(f_{-}\right)=0$. The double points of $f\left(S_{-}^{2}\right)$ may then be labeled $\left\{p_{i}^{+}, p_{i}^{-}\right\}_{i=1}^{k}$ so that $\operatorname{sign}\left(p_{i}^{+}\right)=-\operatorname{sign}\left(p_{i}^{-}\right)$and $n_{i}:=$ $\left|n\left(p_{i}^{+}\right)\right|=\left|n\left(p_{i}^{-}\right)\right|$; consequently, by Lemma 2.4 we may let $f^{-1}\left(p_{i}^{+}\right)=\left\{x_{i}^{+}, y_{i}^{+}\right\}$ and $f^{-1}\left(p_{i}^{-}\right)=\left\{x_{i}^{-}, y_{i}^{-}\right\}$so that if $\gamma_{i}$ is an arc on $S_{-}^{2}$ connecting $x_{i}^{+}$to $x_{i}^{-}$(and missing all other double point preimages) and $\gamma_{i}^{\prime}$ is an $\operatorname{arc}$ on $S_{-}^{2}$ connecting $y_{i}^{+}$to $y_{i}^{-}$ (and missing $\gamma_{i}$ and all other double point preimages), then the loop $f\left(\gamma_{i}\right) \cup f\left(\gamma_{i}^{\prime}\right) \subset$ $f\left(S_{-}^{2}\right)$ is nullhomologous, hence nullhomotopic, in $X_{+}$. Let $U_{i}$ (respectively, $U_{i}^{\prime}$ ) be a neighborhood of $\gamma_{i}$ (respectively, $\gamma_{i}^{\prime}$ ) in $S_{-}^{2}$. The arcs $\left\{\gamma_{i}, \gamma_{i}^{\prime}\right\}_{i=1}^{k}$ and neighborhoods $\left\{U_{i}, U_{i}^{\prime}\right\}_{i=1}^{k}$ may be chosen so that the collection $\left\{U_{i}\right\}_{i=1}^{k} \cup\left\{U_{i}^{\prime}\right\}_{i=1}^{k}$ is mutually disjoint, and so that the resulting Whitney circles $\left\{f\left(\gamma_{i} \cup \gamma_{i}^{\prime}\right)\right\}_{i=1}^{k}$ are mutually disjoint, simple circles in $X_{+}$such that each bounds an immersed, self-transverse Whitney disk $W_{i}$ in $X_{+}$whose interior is transverse to $f\left(S_{-}^{2}\right)$.

Since the two branches $f\left(U_{i}\right)$ and $f\left(U_{i}^{\prime}\right)$ of $f\left(S_{-}^{2}\right)$ meet transversely at $\left\{p_{i}^{+}, p_{i}^{-}\right\}$, there are a pair of smooth vector fields $v_{1}, v_{2}$ on $\partial W_{i}$ such that $v_{1}$ is tangent to $f\left(S_{-}^{2}\right)$ along $\gamma_{i}$ and normal to $f\left(S_{-}^{2}\right)$ along $\gamma_{i}^{\prime}$, while $v_{2}$ is normal to $f\left(S_{-}^{2}\right)$ along $\gamma_{i}$ and tangent to $f\left(S_{-}^{2}\right)$ along $\gamma_{i}^{\prime}$. Such a pair defines a normal framing of $W_{i}$ on the boundary. We say that $\left\{v_{1}, v_{2}\right\}$ is a correct framing of $W_{i}$, and that $W_{i}$ is framed, if the pair extends to a normal framing of $W_{i}$. By boundary twisting $W_{i}$ (see [4, p. 5]) if necessary, at the cost of introducing more interior intersection points with $f\left(S_{-}^{2}\right)$, we can choose the collection of Whitney disks $\left\{W_{i}\right\}_{i=1}^{k}$ such that each is correctly framed.

Let $1 \leq i \leq k$. To each point of intersection $x \in f\left(S_{-}^{2}\right) \cap \operatorname{int} W_{i}$, let $\beta_{x}$ be a loop that first goes along $f\left(S_{-}^{2}\right)$ from its basepoint to $x$, then along $W_{i}$ to $f\left(\gamma_{i}^{\prime}\right) \subset \partial W_{i}$, then back along $f\left(S_{-}^{2}\right)$ to the basepoint of $f\left(S_{-}^{2}\right)$. Further assume that $\beta_{x}$ avoids 


\section{A. Lightfoot}

the respective double points of $f\left(S_{-}^{2}\right)$ and $W_{i}$, and let $m_{i}(x)=\operatorname{lk}\left(f\left(S_{+}^{2}\right), \beta_{x}\right)$. Let

$$
\mathcal{L}_{i}^{-}(x)=n_{i}+n_{i} m_{i}(x)+m_{i}(x) \bmod 2 ;
$$

summing over all such points of intersection, let

$$
\mathcal{L}^{-}\left(W_{i}\right)=\sum_{x \in f\left(S_{-}^{2}\right) \cap \operatorname{int} W_{i}} \mathcal{L}_{i}^{-}(x) \bmod 2 .
$$

Then Li's $\omega_{-}$-invariant applied to $f$ is defined by

$$
\omega_{-}(f)=\sum_{i=1}^{k} \mathcal{L}^{-}\left(W_{i}\right) \quad \bmod 2 .
$$

We record two observations about this definition for later use. The latter is a special case of Proposition 2.1.

Observation 2.5. If $n_{i}$ is odd then $\mathcal{L}^{-}\left(W_{i}\right) \equiv f\left(S_{-}^{2}\right) \cdot \operatorname{int} W_{i}$, while if $n_{i}$ is even then

$$
\mathcal{L}^{-}\left(W_{i}\right) \equiv \sum_{x \in f\left(S_{-}^{2}\right) \cap \operatorname{int} W_{i}} m_{i}(x) .
$$

Observation 2.6. Suppose $x, y \in f\left(S_{-}^{2}\right) \cap \operatorname{int} W_{i}$, and let $\beta$ be a loop that runs from $x$ to $y$ along $f\left(S_{-}^{2}\right)$, then back to $x$ along int $W_{i}$. Further assume that $\beta$ avoids the respective double points of $f\left(S_{-}^{2}\right)$ and $W_{i}$. Then

$$
m_{i}(x)+m_{i}(y) \equiv \operatorname{lk}\left(f\left(S_{+}^{2}\right), \beta\right) .
$$

Now, suppose $f$ is an arbitrary link map with $\sigma_{-}(f)=0$. By standard arguments we may choose a --good link homotopy representative $f^{\prime}$ of $f$, and $\omega(f)$ is defined by setting $\omega_{-}(f)=\omega_{-}\left(f^{\prime}\right)$. By a result in $[13$, this defines an invariant of link homotopy; Theorem 1.1 gives a new proof. By interchanging the roles of $f_{+}$and $f_{-}$(and instead assuming $\sigma_{+}(f)=0$ ), we obtain $\omega_{+}(f) \in \mathbb{Z}_{2}$ similarly, and write $\omega(f)=\left(\omega_{+}(f), \omega_{-}(f)\right)$.

Remark 2.7. Based on similar geometric principles as $\omega$, Schneiderman and Teichner [17] defined a secondary obstruction with respect to the homotopy invariant $\mu$. When adapted to the context of link homotopy, however, their invariant reduces to $\omega$ [13. Theorem 1.3]. Corollary 1.3 then follows from [17, Theorem 2].

\subsection{Surgering tori to 2-spheres}

A common situation to arise in this paper is the following. Suppose we have a torus (punctured torus, respectively) $T$ in the 4-manifold $Y$, on which we wish to perform surgery along a curve so as to turn it into a 2-sphere (2-disk, respectively) whose self-intersection and intersection numbers may be calculated. Our device for doing so is the following lemma, which is similar to [13 Lemma 4.1] and so its proof is omitted. A similar construction may be found at the bottom of [3, p. 86]. 
If $D_{A} \subset A$ is an immersed 2-disk, let $\left.\mu(A)\right|_{D_{A}}$ denote the contribution to $\mu(A)$ due to self-intersection points on $D_{A}$.

Lemma 2.8. Suppose that $Y$ is a codimension-0 submanifold of $S^{4}$ and $\pi_{1}(Y)$ is abelian. Let $B$ be a properly immersed 2-disk or 2-sphere in $Y$, and suppose $T$ is an embedded torus (or punctured torus) in $Y \backslash$ int B. Let $\delta_{0}$ be a simple, non-separating curve on $T$, let $\delta_{1}$ be a normal pushoff of $\delta_{0}$ on $T$ and let $\hat{T}$ denote the annulus on $T$ bounded by $\delta_{0} \cup \delta_{1}$. Suppose there is a map

$$
J: D^{2} \times I \rightarrow Y
$$

such that $J\left(D^{2} \times I\right) \cap T=J\left(\partial D^{2} \times I\right)=\hat{T}$ and $J\left(\partial D^{1} \times\{i\}\right)=\delta_{i}$ for $i=0,1$. Then, after a small perturbation,

$$
S=(T \backslash \operatorname{int} \hat{T}) \bigcup_{\delta_{0} \cup \delta_{1}} J\left(D^{2} \times\{0,1\}\right)
$$

is a properly immersed, self-transverse 2-sphere (or 2-disk, respectively) in $Y$ such that

(i) $\lambda(S, B)=\left(1-\left[\delta_{2}\right]\right) \lambda(D, B)$ in $\mathbb{Z}\left[\pi_{1}(Y)\right]$, and

(ii) $\left.\mu(S) \equiv \mu(S)\right|_{D}+\left.\mu(S)\right|_{D^{\prime}}+\left(D \cdot D^{\prime}\right)\left[\delta_{2}\right]$ in $Q(Y) \bmod 2$,

where $^{\mathrm{b}} D=J\left(D^{2} \times 0\right) \subset S$ is oriented consistently with and shares a whisker with $S, D^{\prime}=J\left(D^{2} \times 1\right)$, and $\delta_{2}$ is a dual curve to each of $\delta_{0}$ and $\delta_{1}$ on $T$ such that $\delta_{2} \cap \hat{T}$ is a simple arc running from $\delta_{0}$ to $\delta_{1}$.

The hypotheses of this lemma will frequently be encountered in the following form. We have $T$ and the nullhomotopic curve $\delta_{0}$, an immersed 2-disk $D \subset Y$ bounded by $\delta_{0}$, and $J$ is a certain homotopy from $D$ to a nearby copy.

\section{Proof of Theorem 1.1}

Let us first outline the steps of the proof. In Sec. 3.1 we recall the notion of an unknotted immersion and show that, up to link homotopy, one component $f\left(S_{-}^{2}\right)$ of a link map $f$ may be assumed to be of this form. We exploit this to construct a collection of mutually disjoint, embedded, framed Whitney disks $\left\{V_{i}\right\}_{i}$ for $f\left(S_{-}^{2}\right)$ with interiors in $S^{4} \backslash f\left(S_{-}^{2}\right)$, such that each has nullhomotopic boundary in the complement of $f\left(S_{+}^{2}\right)$. We show how these disks (along with disks bounded by accessory circles for $\left.f_{-}\right)$can be used to construct 2-sphere generators of $\pi_{2}\left(X_{-}\right)$. The algebraic intersections between $f\left(S_{+}^{2}\right)$ and these 2-spheres are then computed in terms of the intersections between $f\left(S_{+}^{2}\right)$ and the aforementioned disks.

In Sec. 3.2, we surger the disks $\left\{V_{i}\right\}_{i}$ so to exchange their intersections with $f\left(S_{+}^{2}\right)$ for intersections with $f\left(S_{-}^{2}\right)$. In this way, we obtain immersed, framed

\footnotetext{
${ }^{b}$ Note that we shall sometimes exclude curly brackets for a one-point set that occurs in a Cartesian product.
} 


\section{A. Lightfoot}

Whitney disks $\left\{W_{i}\right\}_{i}$ for $f_{-}$in $S^{4} \backslash f\left(S_{+}^{2}\right)$, such that the algebraic intersections between $f\left(S_{-}^{2}\right)$ and $W_{i}$, measured by $\omega_{-}(f)$, are related to the algebraic intersections between $f\left(S_{+}^{2}\right)$ and $V_{i}$.

In Sec. 3.3. we complete the proof by combining the results of these two sections: the intersections between $f\left(S_{+}^{2}\right)$ and generators of $\pi_{2}\left(X_{-}\right)$of the former section are related to $\sigma_{+}(f)$, which by the latter section can be related to $\omega_{-}(f)$.

\subsection{Unknotted immersions and Whitney disks in $X_{-}$}

A notion of unknottedness for surfaces in 4-space was introduced by Hosokawa and Kawauchi in 6]. A connected, closed, orientable surface in $\mathbb{R}^{4}$ is said to be unknotted if it bounds an embedded 3-manifold in $\mathbb{R}^{4}$ obtained by attaching (3-dimensional) 1-handles to a 3-ball. They showed that by attaching (2-dimensional) 1-handles only, any embedded surface in $\mathbb{R}^{4}$ can be made unknotted. Kamada [7] extended their definition to immersed surfaces in $\mathbb{R}^{4}$ and gave a notion of equivalence for such immersions. In that paper, it was shown that an immersed 2 -sphere in $\mathbb{R}^{4}$ can be made equivalent, in this sense, to an unknotted, immersed one by performing (only) finger moves.

It was noted in [13] that we may perform a link homotopy to "unknot" the immersed components of a link map (see Lemma 3.2). The algebraic topology of the complement of an unknotted (immersed) component is greatly simplified, making the computation of the invariants defined in the previous section more tractable.

Let us begin with a precise definition of an unknotted, immersed 2-sphere. To do so, we construct cusp regions which have certain symmetry properties; our justification for these specifications is to follow. Using the moving picture method, Figs.1(a) and1(b) (respectively) illustrate properly immersed, oriented 2-disks $D^{+}$, $D^{-}$(respectively) in $D^{4}$, each with precisely one double point $r^{+}, r^{-}$(respectively) of opposite sign. In those figures, we have indicated coordinates $\left(x_{1}, x_{2}, x_{3}, x_{4}\right)$ of $D^{4}$; our choice of the $x_{2}$-ordinate to represent "time" is a compromise between ease of illustration and ease of subsequent notation.

As suggested by these figures, we construct $D^{ \pm}$so that it has boundary $\partial D^{ \pm}=$ $\partial D^{2} \times 0 \times 0$ and so that it intersects $D^{1} \times 0 \times D^{1} \times D^{1}$ in an arc lying in the plane $D^{1} \times 0 \times D^{1} \times 0$. Further, letting $\theta^{ \pm}$denote the loop on $D^{ \pm}$in this plane that is based at $r^{ \pm}$and oriented as indicated in those figures, we have that the reverse loop $\overline{\theta^{ \pm}}$is given by ${ }^{\mathrm{c}}$

$$
\Sigma \circ \theta^{ \pm}=\overline{\theta^{ \pm}}
$$

${ }^{\mathrm{c}}$ More precisely, we can choose a parameterization $\theta^{ \pm}: I \rightarrow D^{1} \times 0 \times D^{1} \times 0$ and paths $\theta_{i}^{ \pm}: I \rightarrow D^{1}$, $i=1,3$, so that for $t \in I$ we have $\theta^{ \pm}(t)=\left(\theta_{1}^{ \pm}(t), 0, \theta_{3}^{ \pm}(t), 0\right)$ and hence

$$
\Sigma\left(\theta^{ \pm}(t)\right)=\left(-\theta_{1}^{ \pm}(t), 0, \theta_{3}^{ \pm}(t), 0\right)=\overline{\theta^{ \pm}}(t) .
$$




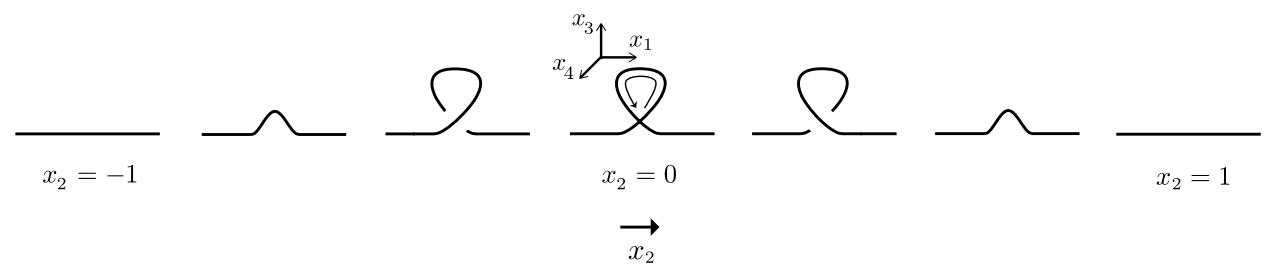

(a) The immersed 2-disk $D^{+}$with one double point $r^{+}$.

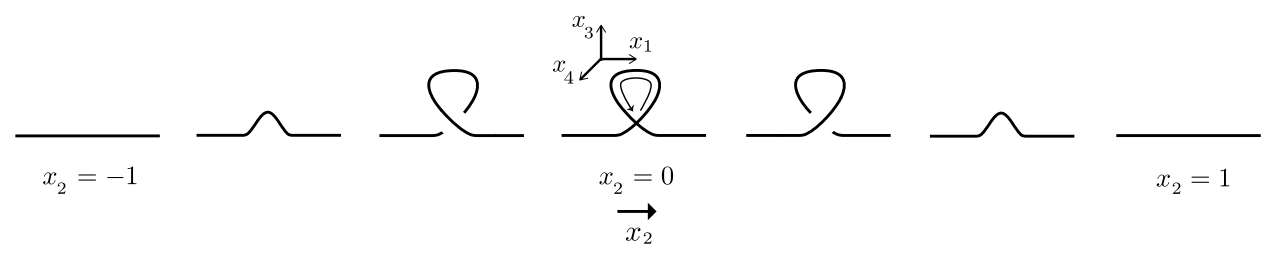

(b) The immersed 2-disk $D^{-}$with one double point $r^{-}$.

Fig. 1.

where $\Sigma$ is the orientation-preserving self-diffeomorphism of $D^{4}$ given by $\left(x_{1}, x_{2}, x_{3}, x_{4}\right) \mapsto\left(-x_{1},-x_{2}, x_{3}, x_{4}\right)$. Lastly, we may suppose that

$$
\Sigma\left(D^{ \pm}\right)=D^{ \pm}
$$

After orienting $D^{4}$ appropriately, the immersed 2-disk $D^{+}\left(D^{-}\right.$, respectively) has a single, positively (negatively, respectively) signed double point $r^{+}\left(r^{-}\right.$, respectively), and $\theta^{ \pm}$is an oriented loop on $D^{ \pm}$based at $r^{ \pm}$which changes branches there. (Note also that in this construction we may suppose that $D^{-}$is the image of $D^{+}$under the orientation-reversing self-diffeomorphism of $D^{4}$ given by $\left(x_{1}, x_{2}, x_{3}, x_{4}\right) \mapsto\left(-x_{1}, x_{2}, x_{3}, x_{4}\right)$.) We call $D^{+}$and $D^{-}$cusps.

Roughly speaking, an unknotted immersion is obtained from an unknotted embedding by "grafting on" cusps of this form. The purpose of the above specifications is so that, by a manoeuvre resembling the Disk Theorem, we may more conveniently move these cusps around on the 2-sphere so that accessory circles of the form $\theta^{ \pm}$are permuted and perhaps reversed as oriented loops.

Formally, let $d \geq 0$, let $\varepsilon:\{1,2, \ldots, d\} \rightarrow\{+,-\}$ be a map which associates a $+\operatorname{sign}$ or a $-\operatorname{sign}$ to each $i \in\{1,2, \ldots, d\}$, and write $\varepsilon_{i}=\varepsilon(i)$. Let $\mathcal{U}$ denote the image of an oriented, unknotted embedding $S^{2} \rightarrow S^{4}$; that is, an embedding that extends to the 3-ball (which is unique up to ambient isotopy). Suppose there are a collection of mutually disjoint, equi-oriented embeddings $b_{i}: D^{4} \rightarrow S^{4}, i=1, \ldots, d$, such that

$$
b_{i}^{-1}(\mathcal{U})=D^{2} \times 0 \times 0
$$

for each $1 \leq i \leq d$. By removing the interiors of the 2-disks $\left\{b_{i}\left(D^{2} \times 0 \times 0\right)\right\}_{i}$ from $\mathcal{U}$ and attaching, for each $1 \leq i \leq d$, the cusp $b_{i}\left(D^{\varepsilon_{i}}\right)$ along $b_{i}\left(\partial D^{2} \times 0 \times 0\right)$, we 


\section{A. Lightfoot}

obtain an unknotted, immersed 2-sphere in $S^{4}$ :

$$
\mathcal{U}_{\varepsilon}=\left[\mathcal{U} \backslash \bigcup_{i=1}^{d} \operatorname{int} b_{i}\left(D^{4}\right)\right] \cup \bigcup_{i=1}^{d} b_{i}\left(D^{\varepsilon_{i}}\right)
$$

Note that we use the function $\varepsilon$ in (3.3) only for convenience of notation in the proofs that follow. Since the embeddings $\left\{b_{i}\right\}_{i}$ can always be relabeled, one sees that the definition of $\mathcal{U}_{\varepsilon}$ depends precisely on the choice of unknotted 2 -sphere $\mathcal{U}$, the embeddings $\left\{b_{i}\right\}_{i}$, and two non-negative integers $d_{+}$and $d_{-}$, where $d_{ \pm}$is the number of $1 \leq i \leq d$ such that $\varepsilon_{i}= \pm$.

The following lemma will allow us to perform an ambient isotopy of $S^{4}$ which carries the model $\mathcal{U}_{\varepsilon}$ back to itself such that accessory circles are permuted (and perhaps reversed in orientation) in a prescribed manner.

Lemma 3.1. Let $\rho$ be a permutation of $\{1,2, \ldots, d\}$. For each $1 \leq i \leq d$, let $\mu_{i} \in\{-1,1\}$. There is an ambient isotopy $\hat{\varphi}: S^{4} \times I \rightarrow S^{4}$ such that $\hat{\varphi}_{1}$ fixes

$$
\mathcal{U}_{\varepsilon} \backslash \bigcup_{i=1}^{d} \operatorname{int} b_{i}\left(D^{4}\right)
$$

set-wise and, for each $1 \leq i \leq d$,

$$
\hat{\varphi}_{1} \circ b_{i}(x, y)=b_{\rho(i)}\left(\mu_{i} x, y\right)
$$

for all $(x, y) \in D^{2} \times D^{2}$. In particular, if $\varepsilon_{\rho(i)}=\varepsilon_{i}$, then $\varphi_{1}\left(\mathcal{U}_{\varepsilon} \cap b_{i}\left(D^{4}\right)\right)=$ $\mathcal{U}_{\varepsilon} \cap b_{\rho(i)}\left(D^{4}\right)$ and

$$
\hat{\varphi}_{1} \circ b_{i} \circ \theta^{\varepsilon_{i}}= \begin{cases}b_{\rho(i)} \circ \theta^{\varepsilon_{i}} & \text { if } \mu_{i}=1, \\ b_{\rho(i)} \circ \overline{\theta^{\varepsilon_{i}}} & \text { if } \mu_{i}=-1 .\end{cases}
$$

The proof consists of using the Disk Theorem [11, Corollary 3.3.7] to transport 4ball neighborhoods of the cusps around the 2-sphere, and is deferred to Appendix A. We proceed instead to apply the lemma to equip one component of a link map, viewed as an immersion into the 4 -sphere, with a particularly convenient collection of mutually disjoint, embedded, framed Whitney disks.

For this purpose, it will be useful to give a particular construction of an unknotted immersion of a 2 -sphere in $S^{4}$ with $d \geq 0$ pairs of opposite-signed double points.

\subsubsection{A model, unknotted immersion}

For $A \subset \mathbb{R}^{3}$ and real numbers $a<b$, write $A[a, b]=A \times[a, b] \subset \mathbb{R}^{3} \times \mathbb{R}$, and $A[a]=A \times a$. Choose an increasing sequence

$$
-1=t_{1}^{-}<t_{2}^{-}<\cdots<t_{d}^{-}<t_{d+1}^{-}=1,
$$

and for each $1 \leq i \leq d$, let $t_{i}^{+}=t_{i+1}^{-}$, write $I_{i}=\left[t_{i}^{-}, t_{i}^{+}\right]$and put $t_{i}=\left(t_{i}^{+}+t_{i}^{-}\right) / 2$. On the unit circle, oriented clockwise, let $x^{+}, x^{-}, y^{-}$and $y^{+}$be distinct, consecutive 


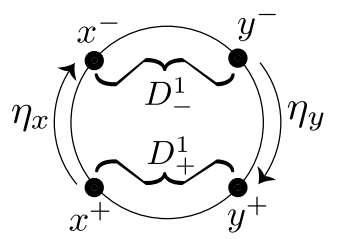

Fig. 2. Labeled arcs on the circle.

points and let $D_{+}^{1}, D_{-}^{1}$ be disjoint (connected) neighborhoods of $\left\{x^{+}, y^{+}\right\},\left\{x^{-}, y^{-}\right\}$, respectively. Let $\hat{\alpha}^{ \pm}: I \rightarrow D_{ \pm}^{1}$ be a path in $D_{ \pm}^{1}$ running from $x^{ \pm}$to $y^{ \pm}$; let $\eta_{x}$ and $\eta_{y}$ be simple paths on $S^{1}$ running $x^{+}$to $x^{-}$and from $y^{-}$to $y^{+}$, respectively. Let $\hat{\eta}_{x}$ and $\hat{\eta}_{y}$ be disjoint neighborhoods of $\eta_{x}$ and $\eta_{y}$ in $S^{1}$, respectively. See Fig. 2,

For each $1 \leq i \leq d$, let $\hat{\Theta}_{i}: D^{1} \rightarrow I_{i}$ be a linear map such that $\hat{\Theta}_{i}(0)=t_{i}$ and $\hat{\Theta}_{i}( \pm 1)=t_{i}^{ \pm}$, and let $\Theta_{i}: D^{3} \times D^{1} \rightarrow D^{3} \times I_{i}$ be the map $\Theta_{i}(x, t)=\left(x, \hat{\Theta}_{i}(t)\right)$. Let $G: S^{1} \times D^{1} \rightarrow D^{3} \times D^{1}$ be an oriented, self-transverse immersion with image as shown in Fig. 3 (ignoring the shadings), with two double-points $p^{ \pm}=G\left(x^{ \pm}, 0\right)=$ $G\left(y^{ \pm}, 0\right)$, such that $G(x, t) \subset D^{3}[t]$ for each $x \in S^{1}$ and $t \in D^{1}$. Then $\alpha^{ \pm}=$ $G\left(\hat{\alpha}^{ \pm} \times 0\right)$ is an oriented loop on $G\left(S^{1} \times D^{1}\right)$ based at $p^{ \pm}$which changes branches there. Note that $G$ is the trace of a regular homotopy between two unknotted circles in $D^{3}$ as illustrated by Fig. [3] By a slight modification of $G$ on $S^{1} \times[-1,-1+\varepsilon]$ and $S^{1} \times[1-\varepsilon, 1]$ for some small $\varepsilon>0$, we may assume $G(x, \pm 1)=(g(x), \pm 1)$ for $x \in S^{1}$, where $g: S^{1} \rightarrow D^{3}$ is an unknot.

For each $1 \leq i \leq d$, define a map $G_{i}: S^{1} \times I_{i} \rightarrow D^{3} \times I_{i}$ by

$$
G_{i}\left(\Theta_{i}(x, t)\right)=\Theta_{i}(G(x, t))
$$

for $(x, t) \in S^{1} \times D^{1}$. Write the 2-sphere as the capped off cylinder

$$
S^{2}=\left(D^{2} \times\{-1,1\}\right) \cup \bigcup_{i=1}^{d} \Theta_{i}\left(S^{1} \times D^{1}\right)
$$

in $D^{3} \times D^{1}$, and define a map $\hat{u}_{d}: S^{2} \rightarrow D^{3} \times D^{1} \subset S^{4}$ by the identity on $D^{2} \times\{-1,1\}$ and by $G_{i}$ on $S^{1} \times I_{i}=\Theta_{i}\left(S^{1} \times D^{1}\right)$.

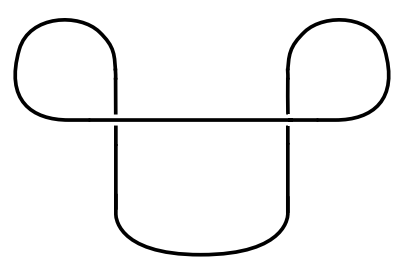

$x_{4}=-1$

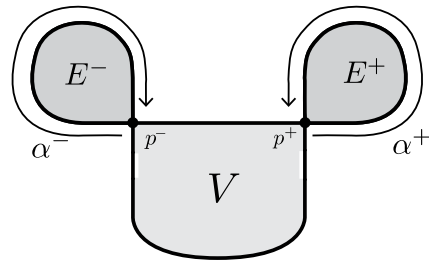

$x_{4}=0$

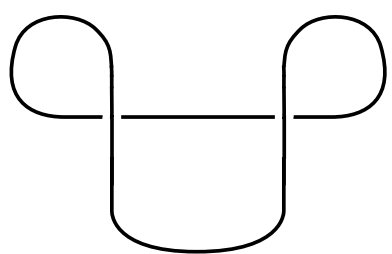

$x_{4}=1$

Fig. 3. A regular homotopy between two unknots. 


\section{A. Lightfoot}

After smoothing corners, $\hat{\mathcal{U}}_{d}=\hat{u}_{d}\left(S^{2}\right)$ is an immersed 2-sphere in $S^{4}$. Let $\alpha_{i}^{ \pm}$ be the oriented loop on $\hat{\mathcal{U}}_{d}$ given by $\alpha_{i}^{ \pm}(s)=\Theta_{i}\left(G\left(\hat{\alpha}^{ \pm}(s), 0\right)\right)=\Theta_{i}\left(\alpha^{ \pm}(s)\right)$ for $s \in I$. Observe that $\alpha_{i}^{ \pm}$is based at the \pm -signed double point $p_{i}^{ \pm}=\Theta_{i}\left(G\left(x^{ \pm}, 0\right)\right)=$ $\Theta_{i}\left(G\left(y^{ \pm}, 0\right)\right)$ and changes branches there.

Referring to Fig. 3, let $V[0] \subset D^{3}[0]\left(V \subset D^{3}\right)$ be the obvious, embedded Whitney disk for the immersed annulus $G\left(S^{1} \times D^{1}\right)$ in $D^{3} \times D^{1}$, bounded by $G\left(\left(\eta_{x} \cup \eta_{y}\right) \times 0\right)$. For arbitrarily small $\varepsilon>0$, by pushing a neighborhood of $G\left(\eta_{y} \times 0\right)$ in $G\left(\hat{\eta}_{y} \times(-\varepsilon, \varepsilon)\right)$ into $D^{3} \times 0$ (as in Fig. 4), we may assume that the Whitney disk is framed: the constant vector field $(0,0,1,0)$ that points out of the page in each hyperplane $\mathbb{R}^{3}\left[t_{0}\right]$ and the constant vector field $(0,0,0,1)$ are a correct framing. Thus, the maps $\left\{\Theta_{i}\right\}_{i=1}^{d}$ carry $V[0]$ to a complete collection of mutually disjoint, embedded, framed Whitney disks $V_{i} \subset \mathbb{R}^{3}\left[t_{i}\right], i=1, \ldots, d$, for $\hat{\mathcal{U}}_{d}$ in $S^{4}$. In particular, the boundary of $V_{i}$ (equipped with an orientation) is given by

$$
\partial V_{i}=\Theta_{i}\left(G\left(\left(\eta_{x} \cup \eta_{y}\right) \times 0\right)\right)=G_{i}\left(\eta_{x} \times 0\right) \cup G_{i}\left(\eta_{y} \times 0\right) .
$$

By [13, Lemma 4.2], we may assume after a link homotopy that one component of a link map is precisely this model of an unknotted immersion.

Lemma 3.2. A link map $f$ is link homotopic to a good link map $g$ such that $g\left(S_{-}^{2}\right)=\hat{\mathcal{U}}_{d}$ for some non-negative integer $d$.

Let $f$ be a good link map such that $f\left(S_{-}^{2}\right)=\hat{\mathcal{U}}_{d}$, and write $X_{ \pm}=S^{4} \backslash f\left(S_{ \pm}^{2}\right)$ as before.

As observed in [13 Lemma 4.3], the complement in $S^{4}$ of an open tubular neighborhood an unknotted, immersed 2-sphere may be equipped with a particularly convenient handlebody decomposition. The decomposition consists of one 0-handle, one 1-handle and one zero-framed 2-handle for each double point of the immersion. The attaching circle of each 2-handle is unknotted in the boundary $S^{3}$ of the 0-handle, and nullhomotopic in the boundary $S^{1} \times S^{2}$ of the union of the 0and 1-handle. In particular, we have the following consequence.

Lemma 3.3. The complement $X_{-}=S^{4} \backslash \hat{U}_{d}$ is homotopy equivalent to the wedge of a circle and $2 d$ two-spheres.
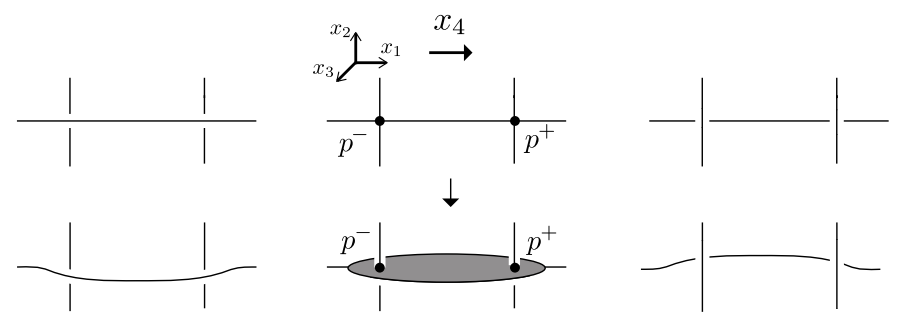

Fig. 4. Placing more of a 2-disk in the "present". 
We next show that the proof of [13, Lemma 4.4] may be generalized to construct representatives of a basis for the free $\mathbb{Z}\left[\pi_{1}\left(X_{-}\right)\right]$-module $\pi_{2}\left(X_{-}\right)$and compute their algebraic intersections with $f\left(S_{+}^{2}\right)$ in terms of the algebraic intersections between $f\left(S_{+}^{2}\right)$ and $\left\{V_{i}\right\}_{i}$.

To simplify notation, assuming the hypotheses of Proposition 3.4. if $A$ is a whiskered 2-disk or 2-sphere in $X_{-}$we write

$$
\lambda_{+}(A)=\lambda\left(f\left(S_{+}^{2}\right), A\right)
$$

in $\mathbb{Z}\left[\pi_{1}\left(X_{-}\right)\right]=\mathbb{Z}\left[s, s^{-1}\right]$, and for a transverse intersection point $x \in f\left(S_{+}^{2}\right) \cap A$ we write $\lambda_{+}(A)[x]=\lambda\left(f\left(S_{+}^{2}\right), A\right)[x]$ in $\pi_{1}\left(X_{-}\right)=\mathbb{Z}\langle s\rangle$.

Proposition 3.4. Let $f$ be a good link map such that $f\left(S_{-}^{2}\right)=\hat{\mathcal{U}}_{d}$. Equip $f\left(S_{+}^{2}\right)$ with a whisker in $X_{-}$and fix an identification of $\pi_{1}\left(X_{-}\right)$with $\mathbb{Z}\langle s\rangle$ so as to write $\mathbb{Z}\left[\pi_{1}\left(X_{-}\right)\right]=\mathbb{Z}\left[s, s^{-1}\right]$. Then $\pi_{2}\left(X_{-}\right) \cong\left(\bigoplus_{i=1}^{d} \mathbb{Z}\right)\left[s, s^{-1}\right]$ and there is a $\mathbb{Z}\left[s, s^{-1}\right]$-basis represented by mutually disjoint, self-transverse, immersed, whiskered 2-spheres $\left\{A_{i}^{+}, A_{i}^{-}\right\}_{i=1}^{d}$ in $X_{-}$with the following properties. For each $1 \leq i \leq d$, there is an integer Laurent polynomial $q_{i}(s) \in \mathbb{Z}\left[s, s^{-1}\right]$ such that

(i) $n_{i}:=q_{i}(1)=\operatorname{lk}\left(f_{+}, \alpha_{i}^{+}\right)$,

(ii) $\lambda\left(A_{i}^{ \pm}, A_{i}^{ \pm}\right)=s+s^{-1} \bmod 2$,

(iii) $\lambda_{+}\left(A_{i}^{+}\right)=(1-s)^{2} q_{i}(s)$, and

(iv) $\lambda_{+}\left(A_{i}^{-}\right)-\lambda_{+}\left(A_{i}^{+}\right)=(1-s)^{2} \lambda_{+}\left(V_{i}^{c}\right)$

in $\mathbb{Z}\left[\pi_{1}\left(X_{-}\right)\right]=\mathbb{Z}\left[s, s^{-1}\right]$, where $V_{i}^{c}$ is a 2 -disk in $X_{-}$obtained from $V_{i}$ by removing a collar in $X_{+}$. Moreover, if for any $1 \leq j \leq d$ and $\varepsilon \in\{-,+\}$ the loop $\alpha_{j}^{\varepsilon}$ bounds a 2-disk in $S^{4}$ that intersects $f\left(S_{+}^{2}\right)$ exactly once, then we may choose $A_{j}^{\varepsilon}$ so that

$$
\lambda_{+}\left(A_{j}^{\varepsilon}\right)=(1-s)^{2} .
$$

While parts (i)-(iii) of Proposition 3.4 may be deduced directly from [13, we provide a complete proof in Appendix B It is roughly summarized as follows.

Near each double point $p_{i}^{ \pm}$of $f\left(S_{-}^{2}\right)=\hat{U}_{d}$ lies a certain torus $T_{i}^{ \pm}$(a copy of the torus $T^{ \pm}$in Fig B.1) such that the collection $\left\{T_{i}^{+}, T_{i}^{-}\right\}_{i=1}^{d}$ represents a basis for $H_{2}\left(X_{-}\right) \cong \mathbb{Z}^{2 d}$. The desired 2-sphere $A_{i}^{ \pm}$is obtained by surgering $T_{i}^{ \pm}$as in Sec. 2.3, there is non-separating curve on $T_{i}^{ \pm}$(resembling the curve $\hat{\delta}^{ \pm}$in Fig. B.2) which bounds a 2-disk $O_{i}^{ \pm}$in $X_{-}$to be described next, and the surgery of $T_{i}^{ \pm}$is performed along $O_{i}^{ \pm}$and a pushoff. The accessory circle $\alpha_{i}^{+}$for $p_{i}^{+}$bounds a 2-disk $E_{i}^{+}$(a copy of the disk $E^{+}$in Fig. 3), and the disk $O_{i}^{+}$is constructed using two copies of $E_{i}^{+}$. On the other hand, the disk $O_{i}^{-}$is constructed using two copies of $E_{i}^{+} \cup V_{i}$ (which is a copy of $E^{+} \cup V$ in Fig. 3). The proposition is obtained upon making this description precise and by applications of Lemma 2.8]

We proceed to apply Lemma 3.1 to prove the following result, which will allow us to surger out the intersections between each $V_{i}$ and $f\left(S_{+}^{2}\right)$ (in exchange for intersections with $f\left(S_{-}^{2}\right)$ ). 


\section{A. Lightfoot}

Proposition 3.5. Let $f$ be a good link map such that $\sigma_{-}(f)=0$ and $f\left(S_{-}^{2}\right)=\hat{\mathcal{U}}_{d}$ for some $d \geq 0$. Then, perhaps after an ambient isotopy, we may assume that $f\left(S_{-}^{2}\right)=\hat{\mathcal{U}}_{d}$ and the (mutually disjoint) embedded Whitney disks $\left\{V_{i}\right\}_{i=1}^{d}$ in $S^{4}$ are framed and satisfy $V_{i} \cdot f\left(S_{+}^{2}\right)=0$ for each $1 \leq i \leq d$.

The proof will occupy the remainder of this section. Recall from the above that on the immersed circle $G\left(S^{1} \times 0\right)$ in $D^{3}[0]$, the arc $G\left(D_{ \pm}^{1} \times 0\right)$ contains the loop $\alpha^{ \pm}=G\left(\hat{\alpha}^{ \pm} \times 0\right)$ in its interior.

Lemma 3.6. Let $d \geq 0$. For each $i \in\{1,2, \ldots, d\}$ let $\mu_{i} \in\{-1,1\}$, and let $\varsigma$ be a permutation of $\{1,2, \ldots, d\}$. There is a 4-ball neighborhood $B_{i}^{ \pm}$of $G_{i}\left(D_{ \pm}^{1} \times S^{1}\right)$ in $S^{4}, i=1, \ldots, d$, and an ambient isotopy $\varphi: S^{4} \times I \rightarrow S^{4}$ such that $\varphi_{1}\left(\hat{\mathcal{U}}_{d}\right)=\hat{\mathcal{U}}_{d}$ and, for each $1 \leq i \leq d$,

(i) $\varphi_{1}$ restricts to the identity on $B_{i}^{+}\left(\right.$so $\left.\varphi_{1} \circ \alpha_{i}^{+}=\alpha_{i}^{+}\right)$,

(ii) $\varphi_{1}$ carries $\left(B_{i}^{-}, G_{i}\left(D_{-}^{1} \times D^{1}\right)\right)$ to $\left(B_{\varsigma(i)}^{-}, G_{\varsigma(i)}\left(D_{-}^{1} \times D^{1}\right)\right)$ and

(iii) $\varphi_{1} \circ \alpha_{i}^{-}= \begin{cases}\alpha_{\varsigma(i)}^{-} & \text {if } \mu_{i}=1, \\ \overline{\alpha_{\varsigma(i)}^{-}} & \text {if } \mu_{i}=-1 .\end{cases}$

Proof. By the construction of $G$, there are disjoint 3-balls $B_{+}^{3}$ and $B_{-}^{3}$ in $D^{3}$ such that $B_{ \pm}^{3}[0]$ is a neighborhood of $E^{ \pm} \subset D^{3}[0]$ and such that there is an orientationpreserving diffeomorphism $\Pi^{ \pm}: D^{4} \rightarrow B_{ \pm}^{3} \times D^{1}$ carrying the cusp $D^{ \pm}$of Sec. 3.1 to $G\left(D_{ \pm}^{1} \times D^{1}\right)$, the double point $r^{ \pm}$to $p^{ \pm}$, and the oriented loop $\theta^{ \pm}$to $\alpha^{ \pm}$.

For each $1 \leq i \leq d$, let $\hat{\mu}_{i} \in\{-,+\}$ denote the sign of $\mu_{i}$, let $\Psi_{i}^{ \pm}$be the orientation-preserving diffeomorphism given by

$$
\Psi_{i}^{ \pm}=\Theta_{i} \circ \Pi^{ \pm}: D^{2} \times D^{2} \rightarrow B_{ \pm}^{3} \times I_{i}
$$

and let $\overline{\Psi_{i}^{ \pm}}=\Psi_{i}^{ \pm} \circ \Sigma$, where $\Sigma$ is the orientation-preserving diffeomorphism of $D^{2} \times D^{2}$ defined in Sec. 3.1 by $\Sigma(x, y)=(-x, y)$. Recalling Eqs. (3.1) and (3.2), observe that $\Psi_{i}^{ \pm}\left(D^{ \pm}\right)=G_{i}\left(D_{ \pm}^{1} \times D^{1}\right)=\overline{\Psi_{i}^{ \pm}}\left(D^{ \pm}\right)$,

$$
\Psi_{i}^{ \pm} \circ \theta^{ \pm}=\alpha_{i}^{ \pm} \text {and } \overline{\Psi_{i}^{ \pm}} \circ \theta^{ \pm}=\overline{\alpha_{i}^{ \pm}} .
$$

Furthermore, by construction, $\hat{\mathcal{U}}_{d}$ is obtained from the unknotted, embedded 2-sphere $\left(S^{1} \times D^{1}\right) \cup\left(D^{2} \times\{ \pm 1\}\right) \subset D^{3} \times D^{1}$ by removing its intersections with the 4-balls $B_{ \pm}^{3} \times I_{i}=\Psi_{i}^{ \pm}\left(D^{4}\right)$, and attaching the cusps $G_{i}\left(D_{ \pm}^{1} \times D^{1}\right)=\Psi_{i}^{ \pm}\left(D^{ \pm}\right)$, for $i=1,2, \ldots, d$. For each $1 \leq i \leq d$, let $b_{2 i}=\Psi_{i}^{+}, b_{2 i-1}=\Psi_{i}^{-}$, and define a permutation $\rho$ on $\{1,2, \ldots, 2 d\}$ by $\rho(2 i)=2 i$ and $\rho(2 i-1)=2 \varsigma(i)-1$. Then Lemma 3.1 yields an ambient isotopy $\varphi: S^{4} \times I \rightarrow S^{4}$ such that $\varphi_{1}$ fixes

$$
\hat{\mathcal{U}}_{d} \backslash \operatorname{int} \bigcup_{i=1}^{d}\left(b_{2 i}\left(D^{4}\right) \cup b_{2 i-1}\left(D^{4}\right)\right)=\hat{\mathcal{U}}_{d} \backslash \operatorname{int} \bigcup_{i=1}^{d}\left(\left(B_{+}^{3} \cup B_{-}^{3}\right) \times I_{i}\right)
$$


set-wise, satisfies

$$
\varphi_{1} \circ \Psi_{i}^{+}=\varphi_{1} \circ b_{2 i}=b_{2 i}=\Psi_{i}^{+}
$$

and

$$
\varphi_{1} \circ \Psi_{i}^{-}(x, y)=\varphi_{1} \circ b_{2 i-1}(x, y)=b_{2 \varsigma(i)-1}\left(\hat{\mu}_{i} x, y\right)=\Psi_{\varsigma(i)}^{-}\left(\hat{\mu}_{i} x, y\right) .
$$

Now, putting $B_{i}^{ \pm}=B^{ \pm} \times I_{i}$, Eq. (3.8) gives part (i) of the lemma; Eq. (3.9) gives part (ii), and part (iii) follows from Eq. (3.6) and by noting that Eq. (3.9) implies $\varphi_{1} \circ \Psi_{i}^{-}=\Psi_{\varsigma(i)}^{-}$if $\mu_{i}=1$ and $\varphi_{1} \circ \Psi_{i}^{-}=\overline{\Psi_{\varsigma(i)}^{-}}$if $\mu_{i}=-1$. Since $\overline{\Psi_{i}^{-}}\left(D^{-}\right)=\Psi_{i}^{-}\left(D^{-}\right)$, $\varphi_{1}$ sends $\bigcup_{i=1}^{d}\left(\Psi_{i}^{+}\left(D^{+}\right) \cup \Psi_{i}^{-}\left(D^{-}\right)\right)$to

$$
\bigcup_{i=1}^{d}\left(\Psi_{i}^{+}\left(D^{+}\right) \cup \Psi_{\varsigma(i)}^{-}\left(D^{-}\right)\right)=\bigcup_{i=1}^{d}\left(\Psi_{i}^{+}\left(D^{+}\right) \cup \Psi_{i}^{-}\left(D^{-}\right)\right),
$$

so $\varphi_{1}\left(\hat{\mathcal{U}}_{d}\right)=\hat{\mathcal{U}}_{d}$ by Eq. (3.7).

We may now perform an ambient isotopy which carries $f\left(S_{-}^{2}\right)=\hat{\mathcal{U}}_{d}$ back to itself in such a way that the accessory circles $\left\{\alpha_{i}^{+}, \alpha_{i}^{-}\right\}_{i=1}^{d}$ are rearranged into canceling pairs with respect to their linking numbers with $f\left(S_{+}^{2}\right)$.

Lemma 3.7. Let $f$ be a link map such that $\sigma_{-}(f)=0$ and $f\left(S_{-}^{2}\right)=\hat{\mathcal{U}}_{d}$ for some $d \geq 0$. Then $f$ is link homotopic (in fact, ambient isotopic) to a link map $g$ such that $g\left(S_{-}^{2}\right)=\hat{\mathcal{U}}_{d}$ and, for each $1 \leq i \leq d$,

$$
\operatorname{lk}\left(\alpha_{i}^{+}, g\left(S_{+}^{2}\right)\right)=\operatorname{lk}\left(\alpha_{i}^{-}, g\left(S_{+}^{2}\right)\right) .
$$

Proof. Since $\sigma_{-}(f)=0$, there is a function $\mu:\{1,2, \ldots, d\} \rightarrow\{-1,1\}$ and a permutation $\varsigma$ on $\{1,2, \ldots, d\}$ such that

$$
\operatorname{lk}\left(\alpha_{i}^{+}, f\left(S_{+}^{2}\right)\right)=\mu_{i} \cdot \operatorname{lk}\left(\alpha_{\varsigma^{-1}(i)}^{-}, f\left(S_{+}^{2}\right)\right)
$$

for each $1 \leq i \leq d$. By Lemma 3.6, there is an ambient isotopy $\varphi: S^{4} \times I \rightarrow S^{4}$ such that $\varphi_{1}\left(\hat{\mathcal{U}}_{d}\right)=\hat{\mathcal{U}}_{d}$ and, for each $1 \leq i \leq d, \varphi_{1} \circ \alpha_{i}^{+}=\alpha_{i}^{+}, \varphi_{1} \circ \alpha_{i}^{-}=\alpha_{\varsigma(i)}^{-}$if $\mu_{i}=1$, and

$$
\varphi_{1} \circ \alpha_{i}^{-}=\overline{\alpha_{\varsigma(i)}^{-}}
$$

if $\mu_{i}=-1$. Then, for each $1 \leq i \leq d$,

$$
\varphi_{1}^{-1}\left(\alpha_{i}^{-}\right)= \begin{cases}\alpha_{\varsigma^{-1}(i)} & \text { if } \mu_{i}=1 \\ \overline{\alpha_{\varsigma^{-1}(i)}} & \text { if } \mu_{i}=-1\end{cases}
$$

and hence

$$
\begin{aligned}
\operatorname{lk}\left(\varphi_{1}^{-1}\left(\alpha_{i}^{-}\right), f\left(S_{+}^{2}\right)\right) & =\mu_{i} \cdot \operatorname{lk}\left(\alpha_{\varsigma^{-1}(i)}, f\left(S_{+}^{2}\right)\right) \\
& =\operatorname{lk}\left(\alpha_{i}^{+}, f\left(S_{+}^{2}\right)\right) .
\end{aligned}
$$




\section{A. Lightfoot}

Thus, taking $g=\varphi_{1} \circ f$, we have

$$
\begin{aligned}
\operatorname{lk}\left(\alpha_{i}^{-}, g\left(S_{+}^{2}\right)\right) & =\operatorname{lk}\left(\varphi_{1}\left(\varphi_{1}^{-1}\left(\alpha_{i}^{-}\right)\right), \varphi_{1}\left(f\left(S_{+}^{2}\right)\right)\right) \\
& =\operatorname{lk}\left(\varphi_{1}^{-1}\left(\alpha_{i}^{-}\right), f\left(S_{+}^{2}\right)\right) \\
& =\operatorname{lk}\left(\alpha_{i}^{+}, f\left(S_{+}^{2}\right)\right) \\
& =\operatorname{lk}\left(\varphi_{1}\left(\alpha_{i}^{+}\right), \varphi_{1}\left(f\left(S_{+}^{2}\right)\right)\right) \\
& =\operatorname{lk}\left(\alpha_{i}^{+}, g\left(S_{+}^{2}\right)\right) .
\end{aligned}
$$

Having established a means to permute the accessory circles of $\hat{\mathcal{U}}_{d}$ in a prescribed way, we may now complete the proof of Proposition 3.5 .

Proof of Proposition [3.5. By Lemma [3.7 we may assume, after an ambient isotopy, that $f\left(S_{-}^{2}\right)=\hat{\mathcal{U}}_{d}$ and the accessory circles $\left\{\alpha_{i}^{+}, \alpha_{i}^{-}\right\}_{i=1}^{d}$ on $f\left(S_{-}^{2}\right)$ satisfy $\operatorname{lk}\left(\alpha_{i}^{+}, f\left(S_{+}^{2}\right)\right)=\operatorname{lk}\left(\alpha_{i}^{-}, f\left(S_{+}^{2}\right)\right)$ for each $1 \leq i \leq d$. Recall the notation of Fig. 2 and that we let $\hat{\eta}_{x}$ and $\hat{\eta}_{y}$ denote disjoint neighborhoods of $\eta_{x}$ and $\eta_{y}$, respectively, on the circle $S^{1}$. For each $1 \leq i \leq d, G_{i}\left(\hat{\eta}_{x} \times\left[-\frac{1}{2}, \frac{1}{2}\right]\right)$ and $G_{i}\left(\hat{\eta}_{y} \times\left[-\frac{1}{2}, \frac{1}{2}\right]\right)$ are embedded 2-disk neighborhoods of $\left\{p_{i}^{+}, p_{i}^{-}\right\}$on $f\left(S_{-}^{2}\right)$ which intersect precisely at these two points, and the accessory circles $\left\{\alpha_{i}^{+}, \alpha_{i}^{-}\right\}$leave along $G_{i}\left(\hat{\eta}_{x} \times\left[-\frac{1}{2}, \frac{1}{2}\right]\right)$ and return along $G_{i}\left(\hat{\eta}_{y} \times\left[-\frac{1}{2}, \frac{1}{2}\right]\right)$. Thus, as the arc $G_{i}\left(\eta_{x} \times 0\right) \subset G_{i}\left(\hat{\eta}_{x} \times\left[-\frac{1}{2}, \frac{1}{2}\right]\right)$ runs from $p^{+}$to $p^{-}$, and the $\operatorname{arc} G_{i}\left(\eta_{y} \times 0\right) \subset G_{i}\left(\hat{\eta}_{y} \times\left[-\frac{1}{2}, \frac{1}{2}\right]\right)$ runs from $p^{+}$to $p^{-}$, by Lemma 2.4 and Eq. (3.5) we have

$$
\begin{aligned}
\left|\operatorname{lk}\left(\partial V_{i}, f\left(S_{+}^{2}\right)\right)\right| & =\left|\operatorname{lk}\left(G_{i}\left(\eta_{x} \times 0\right) \cup G_{i}\left(\eta_{y} \times 0\right), f\left(S_{+}^{2}\right)\right)\right| \\
& =\left|\operatorname{lk}\left(\alpha_{i}^{+}, f\left(S_{+}^{2}\right)\right)-\operatorname{lk}\left(\alpha_{i}^{-}, f\left(S_{+}^{2}\right)\right)\right|=0 .
\end{aligned}
$$

\subsection{Whitney disks in $X_{+}$}

Referring to the notation of Propositions 3.4 and 3.5, we next show that by altering the interiors of the 2-disks $\left\{V_{i}\right\}_{i}$ so to exchange their intersections with $f\left(S_{+}^{2}\right)$ for intersections with $f\left(S_{-}^{2}\right)$, we are able to compute $\omega_{-}$as follows. Recall from Sec. 2 that we use $\equiv$ to denote equivalence modulo 2 , which for integer Laurent polynomials we interpret as equality up to reducing coefficients modulo 2 .

Proposition 3.8. For each $1 \leq i \leq d$, the pair $\left\{p_{i}^{+}, p_{i}^{-}\right\}$of double points of $f\left(S_{-}^{2}\right) \subset X_{+}$may be equipped with a framed, immersed Whitney disk $W_{i}$ in $X_{+}$ such that $\partial W_{i}=\partial V_{i}$. Furthermore, there are integer Laurent polynomials $\left\{u_{i}(s)\right\}_{i=1}^{d}$ such that

$$
\omega_{-}(f) \equiv \sum_{i: n_{i} \text { even }} u_{i}(1)
$$

and for each $1 \leq i \leq d$,

$$
\lambda_{+}\left(V_{i}^{c}\right) \equiv(1+s) u_{i}(s)
$$

where $V_{i}^{c}$ is a 2-disk in $X_{-}$obtained from $V_{i}$ by removing a collar in $X_{+}$. 
Define a ring homomorphism $\phi: \mathbb{Z}\left[s, s^{-1}\right] \rightarrow \mathbb{Z}_{2}$ by

$$
\mathbb{Z}\left[s, s^{-1}\right] \stackrel{\partial}{\longrightarrow} \mathbb{Z}\left[s, s^{-1}\right] \stackrel{s \mapsto 1}{\longrightarrow} \mathbb{Z} \stackrel{\bmod 2}{\longrightarrow} \mathbb{Z}_{2},
$$

where $\partial$ is the formal derivative defined by setting $\partial\left(s^{n}\right)=n s^{n-1}$ (for $n \in \mathbb{Z}$ ) and extending by linearity. For $g(s) \in \mathbb{Z}\left[s, s^{-1}\right]$ we write $\overline{g(s)}=g\left(s^{-1}\right)$. The following properties of $\phi$ are readily verified.

Lemma 3.9. Let $g(s) \in \mathbb{Z}\left[s, s^{-1}\right]$. Then

(i) $\phi(\overline{g(s)}) \equiv \phi(g(s))$,

(ii) $\phi(s \cdot g(s)) \equiv g(1)+\phi(g(s))$, and

(iii) $\phi\left(\left(1+s^{n}\right) g(s)\right) \equiv n \cdot g(1)$.

Moreover, if $h(s) \in \mathbb{Z}\left[s, s^{-1}\right]$ is such that $g(s) \equiv h(s)$, then $\phi(g(s))=\phi(h(s))$.

Let $1 \leq i \leq d$. Since $f\left(S_{+}^{2}\right) \cdot V_{i}=0$, after a small homotopy of $f_{+}$if necessary, we may choose a 2-disk $V_{i}^{c}$ in $X_{-}$obtained from $V_{i}$ by removing a small collar in $X_{+}$so that the intersections between $f\left(S_{+}^{2}\right)$ and int $V_{i}^{c}$ are transverse and may be decomposed into pairs of opposite $\operatorname{sign}\left\{x_{i}^{j}, y_{i}^{j}\right\}_{j=1}^{J_{i}}$ for some $J_{i} \geq 0$ (for any choice of orientation of $S_{+}^{2}$ and $\left.V_{i}^{c}\right)$. For each $1 \leq j \leq J_{i}$, choose a simple path $\alpha_{i}^{j}$ on $f\left(S_{+}^{2}\right)$ (avoiding all double points) from $x_{i}^{j}$ to $y_{i}^{j}$ whose interior is disjoint from $\bigcup_{k=1}^{d} V_{k}$, let $\beta_{i}^{j}$ be a simple path in int $V_{i}^{c}$ from $y_{i}^{j}$ to $x_{i}^{j}$ whose interior misses $f\left(S_{+}^{2}\right)$, and let $\rho_{i}^{j}=\alpha_{i}^{j} \cup \beta_{i}^{j}$. The resulting collection of loops $\left\{\rho_{i}^{j}\right\}_{j=1}^{J_{i}}$ in $X_{-}$may be chosen to be mutually disjoint. For each $1 \leq j \leq J_{i}$, define the $\mathbb{Z}_{2}$-integer

$$
m_{i}^{j}=\operatorname{lk}\left(f\left(S_{-}^{2}\right), \rho_{i}^{j}\right) \bmod 2 .
$$

Note that $m_{i}^{j}$ is independent of the choices made in defining $\rho_{i}^{j}$ because $S_{+}^{2}$ and $V_{i}^{c}$ are simply-connected (cf. Proposition 2.1).

Lemma 3.10. There are integer Laurent polynomials $\left\{u_{i}(s)\right\}_{i=1}^{d}$ in $\mathbb{Z}\left[s, s^{-1}\right]$ such that for each $1 \leq i \leq d$ we have

$$
\lambda_{+}\left(V_{i}^{c}\right) \equiv(1+s) u_{i}(s)
$$

and $u_{i}(1) \equiv \sum_{j=1}^{J_{i}} m_{i}^{j}$

Proof. Choose whiskers connecting $f\left(S_{+}^{2}\right)$ and $\left\{V_{i}^{c}\right\}_{i=1}^{d}$ to the basepoint of $X_{-}$. Let $1 \leq i \leq d$ and $1 \leq j \leq J_{i}$. Since $\rho_{i}^{j}$ is a loop in $X_{-}$that runs from $x_{i}^{j}$ to $y_{i}^{j}$ 


\section{A. Lightfoot}

along $f\left(S_{+}^{2}\right)$, and back to $x_{i}^{j}$ along $V_{i}^{c}$, by Proposition 2.1 we have

$$
\lambda_{+}\left(V_{i}^{c}\right)\left[x_{i}^{j}\right] \cdot\left(\lambda_{+}\left(V_{i}^{c}\right)\left[y_{i}^{j}\right]\right)^{-1}=s^{\hat{m}_{i}^{j}} \in \pi_{1}\left(X_{-}\right),
$$

where $\hat{m}_{i}^{j}$ is an integer such that $\hat{m}_{i}^{j} \equiv \operatorname{lk}\left(f\left(S_{-}^{2}\right), \rho_{i}^{j}\right) \equiv m_{i}^{j}$. Thus

$$
\lambda_{+}\left(V_{i}^{c}\right)\left[x_{i}^{j}\right]=s^{\hat{m}_{i}^{j}} \cdot \lambda_{+}\left(V_{i}^{c}\right)\left[y_{i}^{j}\right],
$$

so the mod 2 contribution to $\lambda_{+}\left(V_{i}^{c}\right)$ due to the pair of intersections $\left\{x_{i}^{j}, y_{i}^{j}\right\}$ is

$$
\lambda_{+}\left(V_{i}^{c}\right)\left[x_{i}^{j}\right]+\lambda_{+}\left(V_{i}^{c}\right)\left[y_{i}^{j}\right] \equiv\left(1+s^{\hat{m}_{i}^{j}}\right) s^{l_{i}^{j}},
$$

for some $l_{i}^{j} \in \mathbb{Z}$. Choosing $u_{i}^{j}(s) \in \mathbb{Z}\left[s, s^{-1}\right]$ such that

$$
(1+s) u_{i}^{j}(s) \equiv\left(1+s^{\hat{m}_{i}^{j}}\right) s^{l_{i}^{j}},
$$

by applying the homomorphism $\phi$ of Lemma 3.9. we see that

$$
u_{i}^{j}(1) \equiv m_{i}^{j} .
$$

Summing over all such pairs $\left\{x_{i}^{j}, y_{i}^{j}\right\}_{j=1}^{J_{i}}$, we have

$$
\lambda_{+}\left(V_{i}^{c}\right) \equiv \sum_{j=1}^{J_{i}}(1+s) u_{i}^{j}(s) \equiv(1+s) u_{i}(s),
$$

where $u_{i}(s)=\sum_{j=1}^{J_{i}} u_{i}^{j}(s)$ satisfies $u_{i}(1) \equiv \sum_{j=1}^{J_{i}} m_{i}^{j}$ by Eq. (3.11).

Let $1 \leq i \leq d$. We now remove the intersections between $V_{i}$ and $f\left(S_{+}^{2}\right)$ by surgering $V_{i}$ along the paths $\left\{\alpha_{i}^{j}\right\}_{j=1}^{J_{i}}$, obtaining an embedded $J_{i}$-genus, once-punctured surface $\hat{V}_{i}$ in $X_{+}$which has interior in $X_{-}$and coincides with $V_{i}$ near the boundary.

Since $f\left(S_{+}^{2}\right)$ is transverse to $V_{i}$, for each $1 \leq j \leq J_{i}$ the restriction of a tubular neighborhood of $f\left(S_{+}^{2}\right)$ to the arc $\alpha_{i}^{j}$ may be identified with a 3 -ball $h_{i}^{j}: D^{1} \times D^{2} \rightarrow$ $X_{-}$such that $h_{i}^{j}\left(D^{1} \times 0\right)=\alpha_{i}^{j}$ and $h_{i}^{j}\left(D^{1} \times D^{2}\right)$ intersects $V_{i}$ in two embedded 2-disks $h_{i}^{j}\left(1 \times D^{2}\right)$ and $h_{i}^{j}\left(-1 \times D^{2}\right)$ neighborhoods of $x_{i}^{j}$ and $y_{i}^{j}$ in $V_{i}$, respectively. Attaching handles to $V_{i}$ along the $\operatorname{arcs} \alpha_{i}^{j}, j=1, \ldots, J_{i}$, yields the surface

$$
\hat{V}_{i}=\left[V_{i} \backslash \bigcup_{j=1}^{J_{i}} \operatorname{int} h_{i}^{j}\left(\partial D^{1} \times D^{2}\right)\right] \bigcup_{j=1}^{J_{i}} \bigcup_{h_{i}^{j}\left(\partial D^{1} \times \partial D^{2}\right)} h_{i}^{j}\left(D^{1} \times \partial D^{2}\right),
$$

whose interior is disjoint from both $f\left(S_{+}^{2}\right)$ and $f\left(S_{-}^{2}\right)$. See Fig. 5 .

Now, for each $1 \leq j \leq J_{i}$, we may assume that $\beta_{i}^{j}$ intersects $h_{i}^{j}\left(1 \times \partial D^{2}\right)$ and $h_{i}^{j}\left(-1 \times \partial D^{2}\right)$ exactly once, at points $\hat{x}_{i}^{j}$ and $\hat{y}_{i}^{j}$, respectively. Let $\hat{\beta}_{i}^{j}$ be the subarc of $\beta_{i}^{j}$ on $\hat{V}_{i}$ running from $\hat{y}_{i}^{j}$ to $\hat{x}_{i}^{j}$, let $\hat{\alpha}_{i}^{j}$ be a path on $h_{j}\left(D^{1} \times \partial D^{2}\right)$ connecting $\hat{x}_{i}^{j}$ to $\hat{y}_{i}^{j}$, and put $\hat{\rho}_{i}^{j}=\hat{\alpha}_{i}^{j} \cup \hat{\beta}_{i}^{j}$. By band-summing $\hat{\alpha}_{i}^{j}$ with meridinal circles of $f\left(S_{+}^{2}\right)$ of the form $h_{j}\left(z \times \partial D^{2}\right)$ (for a point $z$ in int $D^{1}$ ) if necessary, we may assume that $\operatorname{lk}\left(\hat{\rho}_{i}^{j}, f\left(S_{+}^{2}\right)\right)=0$ (see Fig. 5). Hence, as $\pi_{1}\left(X_{+}\right)$is abelian, there is an immersed 2-disk $\hat{Q}_{i}^{j}$ in $X_{+}$bound by $\hat{\rho}_{i}^{j}$. We may further assume that $\hat{Q}_{i}^{j}$ misses a collar of $\partial V_{i}=\partial \hat{V}_{i}$ and is transverse to $f\left(S_{-}^{2}\right)$. 


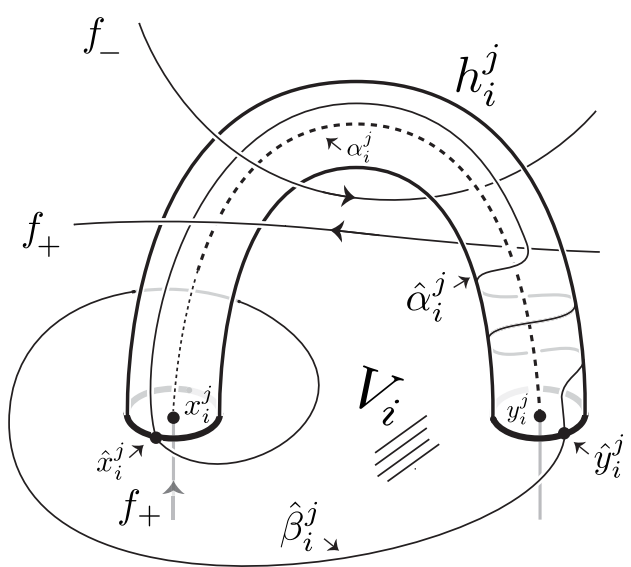

Fig. 5. Surgering $V_{i}$ to remove intersections with $f\left(S_{+}^{2}\right)$ so as to produce the surface $\hat{V}_{i}$ in $X_{+}$.

By boundary twisting $\hat{Q}_{i}^{j}$ along $\hat{\beta}_{i}^{j}$ (and so introducing intersections between the interior of $\hat{Q}_{i}^{j}$ and $\hat{V}_{i}$ ) if necessary we may further assume that a normal section of $\hat{\rho}_{i}^{j}$ that is tangential to $\hat{V}_{i}$ extends to a normal section of $\hat{Q}_{i}^{j}$ in $X_{+}$. Hence there is a normal pushoff $\hat{Q}_{i}^{j}$ of $\hat{Q}_{i}^{j}$ and an annulus $\varrho_{i}^{j}$ on $\hat{V}_{i}$ with boundary $\partial \varrho_{i}^{j}=\partial \hat{Q}_{i}^{j} \cup \partial \hat{Q}_{i}^{j}$ (see Fig. 6). Iterating the construction of Lemma 2.8 we may then surger $\hat{V}_{i}$ along $\hat{\rho}_{i}^{j}$, using $\hat{Q}_{i}^{j}$ and its pushoff $\hat{Q}_{i}^{j}$, for all $1 \leq j \leq J_{i}$, to obtain an immersed 2-disk $W_{i}$ in $X_{+}$such that the framing of $V_{i}$ (which agrees with $W_{i}$ near the boundary) along its boundary extends over $W_{i}$. But $V_{i}$ is a framed Whitney disk for $f\left(S_{-}^{2}\right)$ in $S^{4}$, so $W_{i} \subset X_{+}$is a framed Whitney disk for $f\left(S_{-}^{2}\right)$ in $X_{+} \subset S^{4}$. That is,

$$
W_{i}=\left[\hat{V}_{i} \backslash \operatorname{int} \bigcup_{j=1}^{J_{i}} \varrho_{i}^{j}\right] \bigcup_{j=1}^{J_{i}} \bigcup_{\partial \varrho_{i}^{j}}\left(\hat{Q}_{i}^{j} \cup \hat{Q}_{i}^{j}\right)
$$

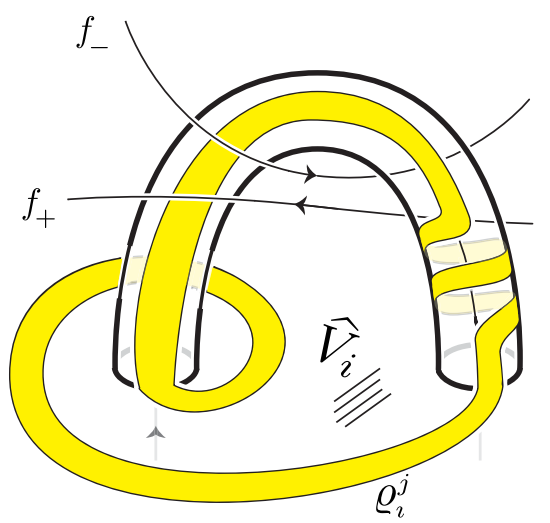

Fig. 6. Towards surgering $\hat{V}_{i}$ to an immersed 2-disk $W_{i}$ in $X_{+}$. 


\section{A. Lightfoot}

is a framed, immersed Whitney disk for the immersion $f_{-}: S^{2} \rightarrow X_{+}$. Let $W_{i}^{c}$ denote the complement in $W_{i}$ of a half-open collar it shares with $V_{i}$ so that $\partial W_{i}^{c}=$ $\partial V_{i}^{c}$ and $f\left(S_{-}^{2}\right)$ intersects $W_{i}^{c}$ in its interior.

The first step in relating $\omega_{-}(f)$ to the intersections between $f\left(S_{+}^{2}\right)$ and the $V_{i}$ 's is the following lemma.

Lemma 3.11. The contribution to $\omega_{-}(f)$ due to intersections between $f\left(S_{-}^{2}\right)$ and the interior of $W_{i}$ is

$$
\mathcal{L}^{-}\left(W_{i}\right) \equiv \begin{cases}0 & \text { if } n_{i} \text { is odd }, \\ \sum_{j=1}^{J_{i}} m_{i}^{j} & \text { if } n_{i} \text { is even } .\end{cases}
$$

Proof. Referring to the constructions preceding the lemma statement, since int $\hat{V}_{i} \subset X_{-}$, the only intersections between int $W_{i}$ and $f\left(S_{-}^{2}\right)$ lie on the immersed 2-disks $\left\{\hat{Q}_{i}^{j}, \hat{Q}_{i}^{j}\right\}_{j=1}^{J_{i}}$. Indeed, since $\hat{Q}_{i}^{j}$ is the pushoff of $\hat{Q}_{i}^{j}$ along a section of its normal bundle that is tangent to the annulus $\varrho_{i}^{j}$ on $\hat{V}_{i}$, there is an immersion of a 3-ball

$$
H_{i}^{j}: D^{2} \times I \rightarrow X_{+}
$$

such that $H_{i}^{j}\left(D^{2} \times 0\right)=\hat{Q}_{i}^{j}, H_{i}^{j}\left(D^{2} \times 1\right)=\hat{Q}_{i}^{j}$ and $H_{i}^{j}\left(S^{1} \times I\right)=\varrho_{i}^{j}$. Furthermore, since $\hat{Q}_{i}^{j}$ is transverse to $f\left(S_{+}^{2}\right)$ we may assume that if we let

$$
K_{i}^{j}=\#\left\{f\left(S_{-}^{2}\right) \cap \hat{Q}_{i}^{j}\right\},
$$

then there are distinct points $x_{k} \in \operatorname{int} D^{2}, 1 \leq k \leq K_{i}^{j}$, such that $f\left(S_{-}^{2}\right)$ intersects $H_{i}^{j}\left(D^{2} \times I\right)$ precisely along the $\operatorname{arcs}\left\{H_{i}^{j}\left(x_{k} \times I\right)\right\}_{k}$. Whence the intersections between $f\left(S_{-}^{2}\right)$ and int $W_{i}$ consist precisely of pairs

$$
\hat{x}_{i}^{j, k}:=H_{i}^{j}\left(x_{k} \times 0\right) \subset \hat{Q}_{i}^{j}
$$

and

$$
x_{i}^{j, k}:=H_{i}^{j}\left(x_{k} \times 1\right) \subset Q_{i}^{j},
$$

for $1 \leq k \leq K_{i}^{j}$. Thus, in particular, if $n_{i}$ is odd then from Observation 2.5 we have $\mathcal{L}^{-}\left(W_{i}\right)=0$.

Suppose now that $n_{i}$ is even. Note that since the loop $\hat{\rho}_{i}^{j}$ on $\hat{V}_{i}$ is freely homotopic in $X_{-}$to $\rho_{i}^{j}$ (to see this, collapse $h_{i}^{j}$ onto its core $\alpha_{i}^{j} \subset f\left(S_{+}^{2}\right)$ in $X_{-}$) we have $\left.\operatorname{lk}\left(f\left(S_{-}^{2}\right), \hat{\rho}_{i}^{j}\right)\right) \equiv m_{i}^{j}$, so

$$
K_{i}^{j} \equiv f\left(S_{-}^{2}\right) \cdot \hat{Q}_{i}^{j} \equiv m_{i}^{j}
$$

We may arrange that there are points $z \in \operatorname{int} D^{1}$ and $d \in S^{1}$ so that the meridinal circle $h_{i}^{j}\left(z \times S^{1}\right)$ of $f\left(S_{+}^{2}\right)$ on $\hat{V}_{i}$ intersects $\varrho_{i}^{j}$ along the arc $H_{i}^{j}(d \times I)$. Let $\iota_{I}$ denote the interval $[0,1]$ oriented from 0 to 1 , and let $\zeta_{i}^{j}$ be the path on $h_{i}^{j}\left(z \times S^{1}\right) \backslash \operatorname{int} \varrho_{i}^{j}$ 
that runs from $H_{i}^{j}(d \times 0)$ to $H_{i}^{j}(d \times 1)$. Then the loop based at $H_{i}^{j}(d \times 1)$ and spanning $h_{i}^{j}\left(z \times S^{1}\right)$ given by

$$
\eta_{i}^{j}:=H_{i}^{j}\left(d \times \overline{\iota_{I}}\right) * \zeta_{i}^{j}
$$

is a meridinal loop of $f\left(S_{+}^{2}\right)$, so $\operatorname{lk}\left(f\left(S_{+}^{2}\right), \eta_{i}^{j}\right) \equiv 1$.

Claim. For fixed $i, j$ : if $n_{i}$ is even then for each $1 \leq k \leq K_{i}^{j}$ the contribution to $\mathcal{L}^{-}\left(W_{i}\right)$ due to the pair $\left\{\hat{x}_{i}^{j, k}, \hat{x}_{i}^{j, k}\right\}$ is

$$
\mathcal{L}_{i}^{-}\left(\hat{x}_{i}^{j, k}\right)+\mathcal{L}_{i}^{-}\left(\dot{x}_{i}^{j, k}\right) \equiv 1
$$

Proof of claim. Let $\gamma_{i}^{j, k}$ be a path in $D^{2}$ connecting $x_{k}$ to $d$. Then

$$
\beta_{i}^{j, k}:=H_{i}^{j}\left(x_{k} \times \overline{\iota_{I}}\right) * H_{i}^{j}\left(\gamma_{i}^{j, k} \times 0\right) * \zeta_{i}^{j} * H_{i}^{j}\left(\overline{\gamma_{i}^{j, k}} \times 1\right)
$$

is a loop that runs from $\dot{x}_{i}^{j, k}$ to $\hat{x}_{i}^{j, k}$ along $H_{i}^{j}\left(x_{k} \times I\right) \subset f\left(S_{-}^{2}\right)$ and then back to $\dot{x}_{i}^{j, k}$ along $W_{i}^{c}$, so by Observations 2.5 and 2.6 we have

$$
\begin{aligned}
\mathcal{L}_{i}^{-}\left(\hat{x}_{i}^{j, k}\right)+\mathcal{L}_{i}^{-}\left(\dot{x}_{i}^{j, k}\right) & \equiv m_{i}\left(\hat{x}_{i}^{j, k}\right)+m_{i}\left(\dot{x}_{i}^{j, k}\right) \\
& \equiv \operatorname{lk}\left(f\left(S_{+}^{2}\right), \beta_{i}^{j, k}\right) .
\end{aligned}
$$

Now, the loop $\beta_{i}^{j, k}$ is homotopic in $X_{+}$to the loop

$$
\left.H_{i}^{j}\left(x_{k} \times \overline{\iota_{I}}\right) * H_{i}^{j}\left(\gamma_{i}^{j, k} \times 0\right) * H_{i}^{j}\left(d \times \iota_{I}\right) * \eta_{i}^{j} * H_{i}^{j} \overline{\left(\gamma_{i}^{j, k}\right.} \times 1\right),
$$

but the loop

$$
\left.H_{i}^{j}\left(x_{k} \times \overline{\iota_{I}}\right) * H_{i}^{j}\left(\gamma_{i}^{j, k} \times 0\right) * H_{i}^{j}\left(d \times \iota_{I}\right) * H_{i}^{j} \overline{\left(\gamma_{i}^{j, k}\right.} \times 1\right)
$$

bounds the 2-disk $H_{i}^{j}\left(\gamma_{i}^{j, k} \times I\right) \subset X_{+}$. Thus, $\beta_{i}^{j, k}$ is homotopic in $X_{+}$to

$$
H_{i}^{j}\left(\gamma_{i}^{j, k} \times 1\right) * \eta_{i}^{j} * H_{i}^{j}\left(\overline{\gamma_{i}^{j, k}} \times 1\right)
$$

and so $\operatorname{lk}\left(f\left(S_{+}^{2}\right), \beta_{i}^{j, k}\right) \equiv \operatorname{lk}\left(f\left(S_{+}^{2}\right), \eta_{i}^{j}\right) \equiv 1$.

Applying the claim to all such pairs of intersections $\left\{\hat{x}_{i}^{j, k}, \hat{x}_{i}^{j, k}\right\}_{k=1}^{K_{i}^{j}}$ between $f\left(S_{+}^{2}\right)$ and $\hat{Q}_{i}^{j} \cup \dot{Q}_{i}^{j}$, over all $1 \leq j \leq J_{i}$, yields the total contribution

$$
\begin{aligned}
\mathcal{L}^{-}\left(W_{i}\right) & \equiv \sum_{x \in f\left(S_{-}^{2}\right) \cap \operatorname{int} W_{i}} \mathcal{L}_{i}^{-}(x) \\
& \equiv \sum_{j=1}^{J_{i}} \sum_{x \in \hat{Q}_{i}^{j} \cup \hat{Q}_{i}^{j}} \mathcal{L}_{i}^{-}(x)
\end{aligned}
$$


A. Lightfoot

$$
\begin{aligned}
& \equiv \sum_{j=1}^{J_{i}} \sum_{k=1}^{K_{i}^{j}}\left(\mathcal{L}_{i}^{-}\left(\hat{x}_{i}^{j, k}\right)+\mathcal{L}_{i}^{-}\left(\hat{x}_{i}^{j, k}\right)\right) \\
& \equiv \sum_{j=1}^{J_{i}} K_{i}^{j} \\
& \equiv \sum_{j=1}^{J_{i}} m_{i}^{j},
\end{aligned}
$$

where the last equality is by Eq. (3.12). This completes the proof of Lemma 3.11

Recalling the definition (2.3) of $\omega_{-}(f)$, by Lemma 3.11 we have

$$
\begin{aligned}
\omega_{-}(f) & \equiv \sum_{i: n_{i} \text { odd }} \mathcal{L}^{-}\left(W_{i}\right)+\sum_{i: n_{i} \text { even }} \mathcal{L}^{-}\left(W_{i}\right) \\
& \equiv \sum_{i: n_{i} \text { even }} \sum_{j=1}^{J_{i}} m_{i}^{j},
\end{aligned}
$$

where $1 \leq i \leq d$. Proposition 3.8 now follows from Lemma 3.10 .

\subsection{Relating $\sigma_{+}$and $\omega_{-}$}

We now bring Kirk's invariant $\sigma_{+}(f)$ into the picture by noting its relationship with the homotopy class of $f\left(S_{+}^{2}\right)$ as an element of $\pi_{2}\left(X_{-}\right)$.

Referring to Proposition [3.4, since $\pi_{2}\left(X_{-}\right)$is generated as a $\mathbb{Z} \pi_{1}\left(X_{-}\right)=$ $\mathbb{Z}\left[s, s^{-1}\right]$-module by the 2 -spheres $\left\{A_{i}^{+}, A_{i}^{-}\right\}_{i=1}^{d}$, there are integer Laurent polynomials $\left\{c_{i}^{+}(s), c_{i}^{-}(s)\right\}_{i=1}^{d}$ such that, as a (whiskered) element of $\pi_{2}\left(X_{-}\right), f\left(S_{+}^{2}\right)$ is given by

$$
f\left(S_{+}^{2}\right)=\sum_{i=1}^{d} c_{i}^{+}(s) A_{i}^{+}+c_{i}^{-}(s) A_{i}^{-} .
$$

By the sesquilinearity of the intersection form $\lambda(\cdot, \cdot)$, we have from Proposition 3.4 that

$$
\begin{aligned}
\lambda\left(f\left(S_{+}^{2}\right), f\left(S_{+}^{2}\right)\right) & \equiv \sum_{i=1}^{d} c_{i}^{+}(s) \overline{c_{i}^{+}(s)} \lambda\left(A_{i}^{+}, A_{i}^{+}\right)+c_{i}^{-}(s) \overline{c_{i}^{-}(s)} \lambda\left(A_{i}^{-}, A_{i}^{-}\right) \\
& \equiv\left(s+s^{-1}\right) \sum_{i=1}^{d}\left[c_{i}^{+}(s) \overline{c_{i}^{+}(s)}+c_{i}^{-}(s) \overline{c_{i}^{-}(s)}\right] .
\end{aligned}
$$

In 8, Kirk showed that $\sigma$ has the following image.

Proposition 3.12 ([8]). If $g$ is a link map, then

$$
\sigma_{+}(g)+\sigma_{-}(g)=a_{0}+\sum_{n=2}^{m} a_{n}\left(n^{2} s-s^{n}\right)
$$


for some integer $m \geq 0$ and integers $a_{0}, a_{2}, a_{3}, \ldots, a_{m}$.

Now, since $\sigma_{-}(f)=0$ and $f$ is a good link map, by Proposition 2.3 we have

$$
\begin{aligned}
\lambda\left(f\left(S_{+}^{2}\right), f\left(S_{+}^{2}\right)\right) & \equiv \sigma_{+}(f)+\overline{\sigma_{+}(f)} \\
& \equiv \sum_{n=2}^{m} a_{n}\left[s^{n}+s^{-n}+n\left(s+s^{-1}\right)\right]
\end{aligned}
$$

for some integers $a_{2}, \ldots, a_{m}$. The following observation about the terms in the right-hand side of this equation will be useful in performing some arithmetic in $\mathbb{Z}_{2}\left[s, s^{-1}\right]$.

Lemma 3.13. Let $n \geq 2$ be an integer. Then

$$
s^{n}+s^{-n}+n\left(s+s^{-1}\right) \equiv(1+s)^{4} r_{n}(s)
$$

for some integer Laurent polynomial $r_{n}(s) \in \mathbb{Z}\left[s, s^{-1}\right]$ such that

$$
r_{n}(1)= \begin{cases}\frac{n}{2} & \text { if } n \text { is even, } \\ 0 & \text { if } n \text { is odd. }\end{cases}
$$

Now, from Eq. (3.13), Proposition 3.4(ii) and the sesquilinearity of $\lambda(\cdot, \cdot)$, we have

$$
\lambda_{+}\left(A_{i}^{ \pm}\right) \equiv c_{i}^{ \pm}(s) \lambda\left(A_{i}^{ \pm}, A_{i}^{ \pm}\right) \equiv c_{i}^{ \pm}(s)\left(s+s^{-1}\right) .
$$

Comparing with Proposition 3.4(iii)-3.4(iv) and Proposition 3.8, we see that there are integer Laurent polynomials $\left\{q_{i}(s), u_{i}(s)\right\}_{i=1}^{d}$ such that

$$
\omega_{-}(f) \equiv \sum_{i: n_{i} \text { even }} u_{i}(1)
$$

and for each $1 \leq i \leq d$,

$$
\begin{aligned}
c_{i}^{+}(s) & \equiv q_{i}(s), \\
c_{i}^{-}(s) & \equiv q_{i}(s)+(1+s) u_{i}(s),
\end{aligned}
$$

where $q_{i}(1) \equiv n_{i}$. Thus, Eq. (3.14) becomes

$$
\begin{aligned}
\lambda\left(f\left(S_{+}^{2}\right), f\left(S_{+}^{2}\right)\right) \\
\equiv\left(s+s^{-1}\right) \sum_{i=1}^{d}\left[q_{i}(s) \overline{q_{i}(s)}+\left(q_{i}(s)+(1+s) u_{i}(s)\right) \overline{\left(q_{i}(s)+(1+s) u_{i}(s)\right)}\right] \\
\equiv\left(s+s^{-1}\right) \sum_{i=1}^{d}\left[(1+s) u_{i}(s) \overline{q_{i}(s)}+\left(1+s^{-1}\right) q_{i}(s) \overline{u_{i}(s)}\right. \\
\left.\quad+(1+s)\left(1+s^{-1}\right) u_{i}(s) \overline{u_{i}(s)}\right] .
\end{aligned}
$$




\section{A. Lightfoot}

Comparing with Eq. 3.15 and applying Lemma 3.13, we then have

$$
\begin{aligned}
\left(s+s^{-1}\right) \sum_{i=1}^{d}\left[(1+s) u_{i}(s) \overline{q_{i}(s)}+\left(1+s^{-1}\right) q_{i}(s) \overline{u_{i}(s)}\right. \\
\left.\quad+(1+s)\left(1+s^{-1}\right) u_{i}(s) \overline{u_{i}(s)}\right] \\
\equiv(1+s)^{4} \sum_{n=2}^{k} a_{n} r_{n}(s) \\
\equiv\left(s+s^{-1}\right)\left(1+s^{-1}\right)^{2} \sum_{n=2}^{k} a_{n} \hat{r}_{n}(s)
\end{aligned}
$$

for some integer Laurent polynomials $\left\{r_{n}(s), \hat{r}_{n}(s)\right\}_{n=2}^{k}$ (here, $\left.\hat{r}_{n}(s)=s^{3} r_{n}(s)\right)$ such that $\hat{r}_{n}(1)=r_{n}(1)=n / 2$ if $n$ is even, and $\hat{r}_{n}(1)=0$ if $n$ is odd. Since $\mathbb{Z}_{2}\left[s, s^{-1}\right]$ is an integral domain, we may divide both sides of Eq. (3.17) by $\left(s+s^{-1}\right)\left(1+s^{-1}\right)$ to obtain

$$
\left(1+s^{-1}\right) \sum_{n=2}^{k} a_{n} \hat{r}_{n}(s) \equiv \sum_{i=1}^{d} u_{i}(s) \overline{q_{i}(s)} s+q_{i}(s) \overline{u_{i}(s)}+(1+s) u_{i}(s) \overline{u_{i}(s)} .
$$

Applying the homomorphism $\phi$ of Lemma 3.9 to both sides of Eq. (3.19) then yields the following equality in $\mathbb{Z}_{2}$ :

$$
\begin{aligned}
\sum_{n=2}^{k} a_{n} \hat{r}_{n}(1) & \equiv \sum_{i=1}^{d}\left[\overline{q_{i}(1)} u_{i}(1)+\phi\left(u_{i}(s) \overline{q_{i}(s)}\right)+\phi\left(q_{i}(s) \overline{u_{i}(s)}\right)+u_{i}(1) \overline{u_{i}(1)}\right] \\
& \equiv \sum_{i=1}^{d} q_{i}(1) u_{i}(1)+u_{i}(1) \\
& \equiv \sum_{i=1}^{d} u_{i}(1)\left(n_{i}+1\right) \equiv \sum_{i: n_{i} \text { is even }} u_{i}(1) .
\end{aligned}
$$

Thus, as $\hat{r}_{n}(1) \equiv 1$ if and only if $n$ is even and $n / 2 \equiv 1$ (i.e. $n=2 \bmod 4$ ), from Eq. (3.16) we have

$$
\omega_{-}(f) \equiv \sum_{n}\left\{a_{n}: n=2 \bmod 4\right\}
$$

completing the proof of Theorem 1.1 .

\section{Appendix A. Proof of Lemma 3.1}

We break the proof into the following lemmas.

Lemma A.1. Fix an orientation of $S^{2}$. Let $\hat{b}_{1}, \hat{b}_{2}: D^{2} \rightarrow S^{2}$ be a pair of mutually disjoint, equi-oriented embeddings. Let $N_{1}, N$ be 2-disk neighborhoods of $\hat{b}_{1}\left(D^{2}\right)$ and 
$\hat{b}_{1}\left(D^{2}\right) \cup \hat{b}_{2}\left(D^{2}\right)$ in $S^{2}$, respectively.

(i) There is an ambient isotopy $g: S^{2} \times I \rightarrow S^{2}$ with support on $N_{1}$ such that $h_{1} \circ \hat{b}_{1}(x, y)=\hat{b}_{1}(-x,-y)$ for $(x, y) \in D^{2}$.

(ii) There is an ambient isotopy $h: S^{2} \times I \rightarrow S^{2}$ with support on $N$ such that $h_{1} \circ \hat{b}_{1}=\hat{b}_{2}$ and $h_{1} \circ \hat{b}_{2}=\hat{b}_{1}$.

Proof. We prove (ii) only; (i) is easier (note that the transformation from $D^{2}=$ $D^{1} \times D^{1}$ to itself given by $(x, y) \mapsto(-x,-y)$ is orientation-preserving).

Let $\hat{N}$ be a 2-disk neighborhood of $\hat{b}_{1}\left(D^{2}\right) \cup \hat{b}_{2}\left(D^{2}\right)$ in the interior of $N$, and choose a collar $c: \partial \hat{N} \times I \rightarrow N$ of $N$ such that $c(x, 0)=x$ for $x \in \partial \hat{N}$ and $c(\partial \hat{N} \times 1)=\partial N$. Since the embeddings $\hat{b}_{1}$ and $\hat{b}_{2}$ are equi-oriented, by the Disk Theorem [11, Corollary 3.3.7] and the Isotopy Extension Theorem [11, Theorem 2.5.2], there is an ambient isotopy $\hat{h}: \hat{N} \times I \rightarrow \hat{N}$ such that $\hat{h}_{1} \circ \hat{b}_{1}=\hat{\hat{b}}_{2}$ and $\hat{h}_{1} \circ \hat{b}_{2}=\hat{b}_{1}$. Choose a smooth function $m: I \rightarrow I$ satisfying $m(0)=1$ and $m(1)=0$, and define $h: S^{2} \times I \rightarrow S^{2}$ as follows. For each $t \in I$, let $h_{t}=\hat{h}_{t}$ on $\hat{N}$, let

$$
h(c(x, s), t)=c(\hat{h}(x, m(s) t), s)
$$

for $(x, s) \in \partial \hat{N} \times I$, and let $h_{t}=\mathrm{id}_{S^{2}}$ elsewhere. It is readily verified that $h_{0}=\mathrm{id}_{S^{2}}$, and that for each $t \in I$, the map $h_{t}$ is well-defined on $\partial \hat{N}=c(d \hat{N} \times 0)$ and constant on the complement of $S^{2} \backslash \operatorname{int} N$.

We extend these isotopies to $S^{4}$ as follows.

Lemma A.2. Suppose that $b_{1}, b_{2}: D^{4} \rightarrow S^{4}$ are a pair of equi-oriented embeddings with mutually disjoint images such that, if $S^{2} \subset S^{4}$ denotes the standard embedding, we have $b_{i}^{-1}\left(S^{2}\right)=D^{2} \times 0 \times 0$ for $i=1,2$. Let $N_{1}, N$ be 2-disk neighborhoods of $b_{1}\left(D^{4}\right) \cap S^{2}$ and $\left(b_{1}\left(D^{4}\right) \cup b_{2}\left(D^{4}\right)\right) \cap S^{2}$ in $S^{2}$, respectively.

(i) There is an ambient isotopy $F: S^{4} \times I \rightarrow S^{4}$ with support on an arbitrarily small 4-ball neighborhood of $b_{1}\left(D^{4}\right) \cup N_{1}$ such that $F_{1}$ fixes $S^{2}$ set-wise, and $F_{1}\left(b_{1}(x, y)\right)=b_{1}(-x, y)$ for $(x, y) \in D^{2} \times D^{2}$.

(ii) There is an ambient isotopy $H: S^{4} \times I \rightarrow S^{4}$ with support on an arbitrarily small 4-ball neighborhood of $b_{1}\left(D^{4}\right) \cup b_{2}\left(D^{4}\right) \cup N$ such that $H_{1}$ fixes

$$
S^{2} \backslash \operatorname{int}\left(b_{1}\left(D^{4}\right) \cup b_{2}\left(D^{4}\right)\right)
$$

set-wise, and $H_{1} \circ b_{1}=b_{2}$ and $H_{1} \circ b_{2}=b_{1}$.

Proof. We prove (ii) only; (i) is an analogous application of part (i) of Lemma A.1. Denote the closed 2-disk in $\mathbb{R}^{2}$ of radius $\frac{1}{2}$ by $\hat{D}^{2}$. Since for $i=1,2, b_{i}\left(D^{4}\right)$ intersects the standard 2-sphere along $b_{i}\left(D^{2} \times 0 \times 0\right)$, we may identify a tubular neighborhood of $S^{2}=S^{2} \times 0 \times 0$ with $S^{2} \times D^{2}$ so that there are equi-oriented, disjoint embeddings 


\section{A. Lightfoot}

$\hat{b}_{i}: D^{2} \rightarrow b_{i}\left(D^{4}\right) \cap S^{2}$ such that $b_{i}\left(D^{4}\right)=\hat{b}_{i}\left(D^{2}\right) \times \hat{D}^{2}$ and $b_{i}$ is given by $b_{i}(x, y)=$ $\left(\hat{b}_{i}(x), \frac{1}{2} y\right)$ for $(x, y) \in D^{2} \times D^{2}$.

By Lemma A.1(ii), there is an ambient isotopy $h: S^{2} \times I \rightarrow S^{2}$ with support on $N$ such that $h_{1} \circ \hat{b}_{1}=\hat{b}_{2}$ and $h_{1} \circ \hat{b}_{2}=\hat{b}_{1}$; in particular, $h_{1}$ fixes $N \backslash \operatorname{int}\left(\hat{b}_{1}\left(D^{2}\right) \cup\right.$ $\left.\hat{b}_{2}\left(D^{2}\right)\right)$ set-wise. We construct an isotopy $H$ of $N \times D^{2} \subset S^{2} \times D^{2}$ such that, for each $t \in I$ :

(1) $\left.H_{t}\right|_{\partial\left(N \times D^{2}\right)}=\operatorname{id}_{\partial\left(N \times D^{2}\right)}$,

(2) $H_{t}(x, y)=\left(h_{t}(x), y\right)$ for all $x \in N, y \in \hat{D}^{2}$, and

(3) $H_{1} \circ b_{1}=b_{2}$ and $H_{1} \circ b_{2}=b_{1}$.

Choose a smooth function $m: I \rightarrow I$ such that $m(1)=0$ and $m(s)=1$ for all $s \in\left[\frac{1}{2}, 1\right]$. For each $t \in I$ and $(x, y) \in N \times D^{2}$, let $H_{t}(x, y)=\left(h_{m(|y|) t}(x), y\right)$, where $|\cdot|$ denotes the Euclidean norm on $D^{2}$. Note that on $N \times D^{2}, H_{t}$ has inverse given by $H_{t}^{-1}(x, y)=\left(h_{m(|y|) t}^{-1}(x), y\right)$. To verify (1), observe that for $x \in \partial N$ and $y \in D^{2}$ we have $\left(h_{m(|y|) t}(x), y\right)=(x, y)$; for $x \in N$ and $y \in \partial D^{2}$, we have $\left(h_{m(|y|) t}(x), y\right)=\left(h_{0}(x), y\right)=(x, y)$. To verify $(2)$, observe that if $y \in \hat{D}^{2}$ then $m(|y|)=1$ and so $\left(h_{m(|y|) t}(x), y\right)=\left(h_{t}(x), y\right)$. Regarding $(3)$, for $(x, y) \in D^{2} \times D^{2}$ we have

$$
H_{1}\left(b_{1}(x, y)\right)=H_{1}\left(\hat{b}_{1}(x), \frac{1}{2} y\right)=\left(h_{1}\left(\hat{b}_{1}(x)\right), \frac{1}{2} y\right)=\left(\hat{b}_{2}(x), \frac{1}{2} y\right)=b_{2}(x, y)
$$

and we have $H_{1} \circ b_{2}=b_{1}$ similarly.

Now, by (1), we may extend $H$ to an isotopy of $S^{4}$ that is constant on the complement of $N \times D^{2}$. Since $h_{1}$ fixes $N \backslash \operatorname{int}\left(\hat{b}_{1}\left(D^{2}\right) \cup \hat{b}_{2}\left(D^{2}\right)\right)=\left[N \backslash \operatorname{int}\left(\hat{b}_{1}\left(D^{2}\right) \cup\right.\right.$ $\left.\left.\hat{b}_{2}\left(D^{2}\right)\right)\right] \times 0$ set-wise, so does $H_{1}$ by property $(2)$; hence $H_{1}$ fixes $S^{2}$ (since $H_{1}$ is the identity outside $N^{2} \times D^{2}$ ).

\section{We may now prove Lemma 3.1 .}

Proof of Lemma 3.1. Choose an orientation-preserving diffeomorphism $\Phi: S^{4} \rightarrow$ $S^{4}$ that takes $\mathcal{U}$ to the standard embedding $S^{2} \subset S^{4}$; then $b_{i}^{\prime}=\Phi \circ b_{i}, i=1, \ldots, d$, is a collection of mutually disjoint, equi-oriented embeddings $D^{4} \rightarrow S^{4}$ whose images intersect $S^{2}$ precisely along $b_{i}^{\prime}\left(D^{2} \times 0 \times 0\right)$, respectively.

If $\rho$ is non-trivial, write it as a product of non-trivial transpositions $\rho=$ $\tau_{1} \tau_{2} \ldots \tau_{n}$ for some $n \geq 1$. For each $1 \leq k \leq n$, write $\tau_{k}=\left(a_{k} b_{k}\right)$ for some $a_{k}, b_{k} \in$ $\{1,2, \ldots, d\}$, and let $N^{(k)}$ be a 2-disk neighborhood of $\left(b_{a_{k}}^{\prime}\left(D^{4}\right) \cup b_{b_{k}}^{\prime}\left(D^{4}\right)\right) \cap S^{2}$ in $S^{2} \backslash \operatorname{int} \bigcup_{i \neq a_{k}, b_{k}} b_{i}^{\prime}\left(D^{4}\right)$. By Lemma A.2(ii), there is an ambient isotopy $H^{(k)}$ : $S^{4} \times I \rightarrow S^{4}$ with support on $N^{(k)} \times D^{2}$ in $S^{4} \backslash \operatorname{int} \bigcup i \neq a_{k}, b_{k} b_{i}^{\prime}\left(D^{4}\right)$ such that $H_{1}^{(k)}$ fixes

$$
S^{2} \backslash \operatorname{int}\left(b_{a_{k}}\left(D^{4}\right) \cup b_{b_{k}}\left(D^{4}\right)\right)
$$


set-wise and is such that $H_{1}^{(k)} \circ b_{i}^{\prime}=b_{\tau_{k}(i)}^{\prime}$ for $i=a_{k}, b_{k}$. Define $H: S^{4} \times I \rightarrow S^{4}$ by

$$
H(x, t)=H^{(k)}\left(x, n\left(t-\frac{k-1}{n}\right)\right)
$$

for $x \in S^{4}$ and $t \in\left[\frac{k-1}{n}, \frac{k}{n}\right]$, where $k=1,2, \ldots, n$. Then $H$ is an ambient isotopy which fixes

$$
S^{2} \backslash \operatorname{int} \bigcup_{i=1}^{d} b_{i}^{\prime}\left(D^{4}\right)
$$

set-wise and satisfies

$$
H_{1} \circ b_{i}^{\prime}=b_{\rho(i)}^{\prime}
$$

for each $1 \leq i \leq d$. Now, by Lemma A.2(i), for each $1 \leq i \leq d$ there is an ambient isotopy $F^{(i)}: S^{4} \times I \rightarrow S^{4}$ with support on a 4-ball neighborhood of $N^{(\rho(i))}$ in $S^{4} \backslash \operatorname{int} \bigcup_{k \neq \rho(i)} b_{k}^{\prime}\left(D^{4}\right)$ such that $F_{1}^{(i)}$ fixes $S^{2}$ set-wise and is such that

$$
F_{1}^{(i)} \circ b_{\rho(i)}^{\prime}(x, y)=b_{\rho(i)}^{\prime}\left(\mu_{i} x, y\right)
$$

for $(x, y) \in D^{2} \times D^{2}$. Define $F: S^{4} \times I \rightarrow S^{4}$ by

$$
F(x, t)=F^{(i)}\left(x, d\left(t-\frac{i-1}{d}\right)\right)
$$

for $x \in S^{4}$ and $t \in\left[\frac{i-1}{d}, \frac{i}{d}\right]$, where $i=1,2, \ldots, d$. Then $F$ is an ambient isotopy which fixes $S^{2}$ set-wise and satisfies

$$
F_{1} \circ b_{\rho(i)}^{\prime}(x, y)=b_{\rho(i)}^{\prime}\left(\mu_{i} x, y\right)
$$

for $(x, y) \in D^{2} \times D^{2}$. Thus, if $K: S^{4} \times I \rightarrow S^{4}$ is the ambient isotopy defined by $H_{2 t}$ for $t \in\left[0, \frac{1}{2}\right]$ and $F_{2 t-1}$ for $t \in\left[\frac{1}{2}, 1\right]$, then $\hat{\varphi}_{t}=\Phi^{-1} \circ K_{t} \circ \Phi$ is the required isotopy.

\section{Appendix B. Proof of Proposition [3.4}

Recalling the notation of (3.1.1), for each $1 \leq i \leq d$ the double points $p_{i}^{+}$and $p_{i}^{-}$of $f\left(S_{-}^{2}\right)$ lie in $D^{3}\left[t_{i}\right]$ and $f\left(S_{-}^{2}\right)$ intersects the 4 -ball $D^{3}\left[t_{i}^{-}, t_{i}^{+}\right] \subset D^{3} \times D^{1}$ precisely along $\Theta_{i}\left(G\left(S^{1} \times D^{1}\right)\right)$. In what follows, we shall denote $\hat{G}=G\left(S^{1} \times D^{1}\right)$; note that for $t \in D^{1}, \hat{G} \cap\left(D^{3}[t]\right)=G\left(S^{1} \times t\right)$ and $\Theta_{i}\left(G\left(S^{1} \times t\right)\right)=f\left(S_{-}^{2}\right) \cap\left(D^{3}\left[\hat{\Theta}_{i}(t)\right]\right)$. Observe that by the construction of the annulus $G$ we may assume there are real numbers $-1<a^{-}<b^{-}<0<a^{+}<b^{+}<1$ such that $G\left(S^{1} \times\left[a^{ \pm}, b^{ \pm}\right]\right)=G_{0}\left[a^{ \pm}, b^{ \pm}\right]$ for some (embedded circle) $G_{0} \subset D^{3}$.

Let $E^{ \pm}$denote the 2-disk bounded by $\alpha^{ \pm}$in $D^{3}[0]$ as in Fig. 3, and choose a 3-ball $N^{ \pm}$so that $N^{ \pm}[-1,1]$ is a 4-ball neighborhood of $p^{ \pm}$and $\Theta_{i}\left(N^{ \pm}[-1,1]\right)$ is disjoint from $f\left(S_{+}^{2}\right)$. There is an embedded torus $T^{ \pm}$in $N^{ \pm}[-1,1] \backslash \hat{G}$ that intersects $E^{ \pm}$ 

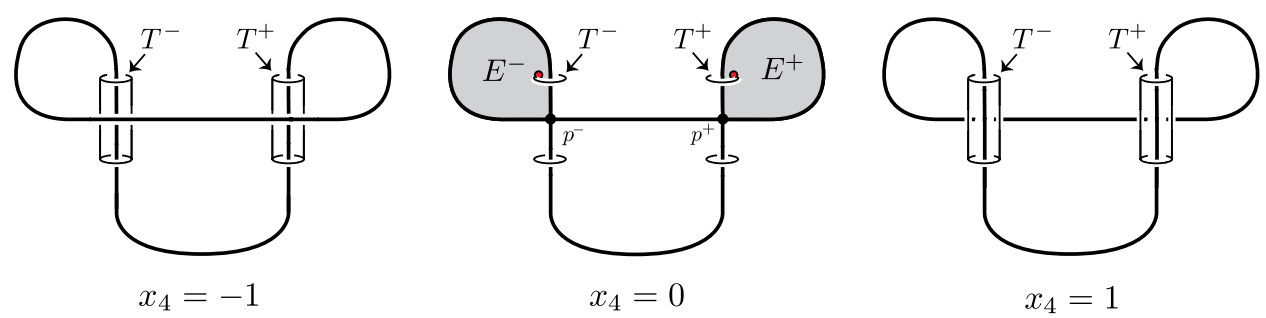

Fig. B.1. Associated to each double point is a linking torus and a disk bound by an accessory circle.

exactly once; see Fig. B.1 for an illustration of $T^{+}$and $T^{-}$in $D^{3} \times D^{1}$. The torus $T^{ \pm}$ appears as a cylinder in each of 3-balls $N^{ \pm}[-1]$ and $N^{ \pm}[1]$, and as a pair of circles in $N^{ \pm}[t]$ for $t \in(-1,1)$. For each $1 \leq i \leq d$, let $T_{i}^{ \pm}=\Theta_{i}\left(T^{ \pm}\right) \subset N^{ \pm}\left[t_{i}^{-}, t_{i}^{+}\right] \backslash f\left(S_{-}^{2}\right)$. By Alexander duality, the linking pairing

$$
H_{2}\left(X_{-}\right) \times H_{1}\left(f\left(S_{-}^{2}\right)\right) \rightarrow \mathbb{Z}
$$

defined by $(R, v) \mapsto R \cdot \Upsilon$, where $v=\partial \Upsilon \subset S^{4}$, is nondegenerate. Thus, as the loops $\left\{\alpha_{i}^{+}, \alpha_{i}^{-}\right\}_{i=1}^{d}$ represent a basis for $H_{1}\left(f\left(S_{-}^{2}\right)\right) \cong \mathbb{Z}^{2 d}$, we have that $H_{2}\left(X_{-}\right) \cong \mathbb{Z}^{2 d}$ and (after orienting) the so-called linking tori $\left\{T_{i}^{+}, T_{i}^{-}\right\}_{i=1}^{d}$ represent a basis.

We proceed to apply the construction of Sec. [2.3] (twice, successively) to turn these tori into 2-spheres.

Let $\widehat{\Delta}^{ \pm} \subset N^{ \pm}$be the embedded 2-disk so that $\widehat{\Delta}^{ \pm}\left[a^{ \pm}\right]$appears in $N^{ \pm}\left[a^{ \pm}\right]$as in Fig. B.2 and let $\hat{\delta}^{ \pm}=\partial \widehat{\Delta}^{ \pm}$. The disk $\widehat{\Delta}^{ \pm}\left[a^{ \pm}\right]$intersects $\hat{G}$ at two points, which are the endpoints of an arc of the form $\gamma^{ \pm}\left[a^{ \pm}\right]\left(\gamma^{ \pm} \subset G_{0}\right)$ in $G\left(S^{1} \times a^{ \pm}\right)$, also illustrated.

In Fig. B.2, we have also illustrated in $D^{3}\left[a^{ \pm}\right]$the restriction of a tubular neighborhood of $\hat{G}$ to $\gamma^{ \pm}\left[a^{ \pm}\right]$, which we may write in the form $\left(\gamma^{ \pm} \times D^{2}\right)\left[a^{ \pm}\right]$and choose so that $\Theta_{i}$ carries $\left(\gamma^{ \pm} \times D^{2}\right)\left[a^{ \pm}\right]$to a tubular neighborhood of $f\left(S_{-}^{2}\right)$ restricted to $\Theta_{i}\left(\gamma^{ \pm}\left[a^{ \pm}\right]\right)$in $S^{4} \backslash f\left(S_{+}^{2}\right)$. Let $\Delta^{ \pm}$be the embedded punctured torus in $D^{3}$ obtained
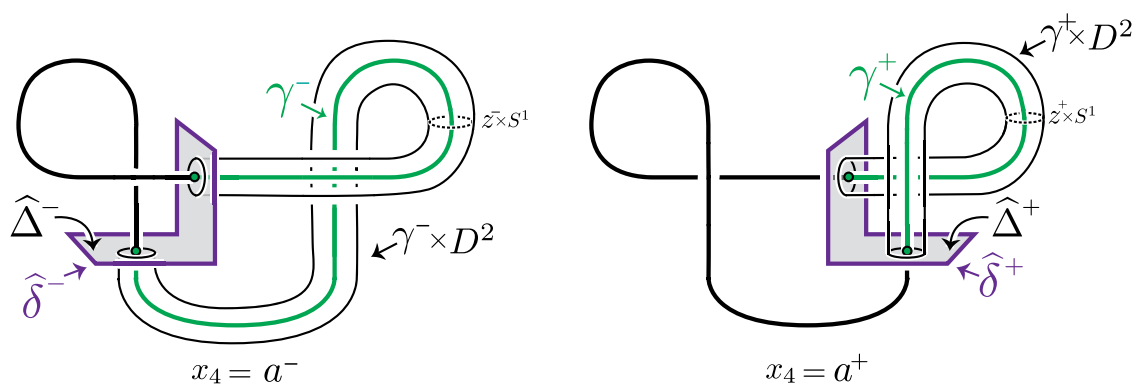

Fig. B.2. The first step to turning $\hat{\Delta}^{ \pm}\left[a^{ \pm}\right]$into a 2-disk missing $\hat{G}$ with a view to applying Lemma 2.8 forming the punctured torus $\Delta^{ \pm}\left[a^{ \pm}\right]$. 

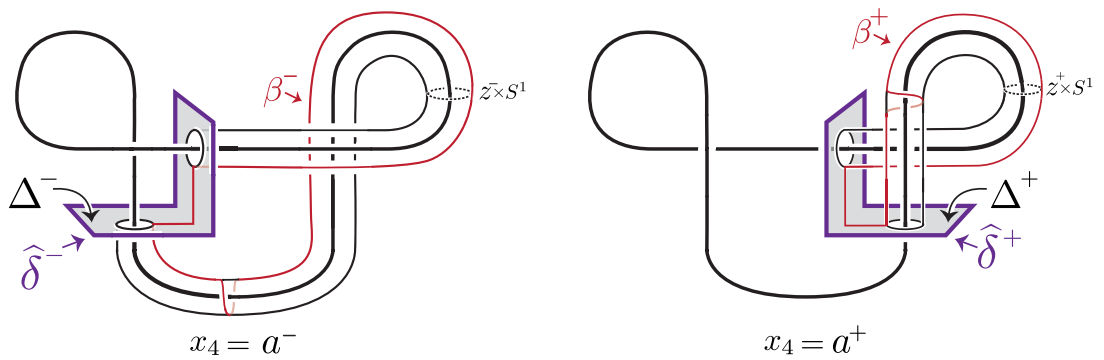

Fig. B.3. The second step: choosing a closed curve on $\Delta^{ \pm}\left[a^{ \pm}\right]$bounding a 2-disk in the complement of $\hat{G}$.

from $\hat{\Delta}^{ \pm}$by attaching a 1-handle along $\gamma^{ \pm}$; that is, let

$$
\Delta^{ \pm}=\left[\hat{\Delta}^{ \pm} \backslash\left(\partial \gamma^{ \pm} \times \operatorname{int} D^{2}\right)\right] \underset{\partial \gamma^{ \pm} \times S^{1}}{\bigcup}\left(\gamma^{ \pm} \times S^{1}\right) .
$$

Note that $\Delta^{ \pm}$has boundary $\hat{\delta}^{ \pm}$.

Let $\beta^{ \pm}$be a loop on $\Delta^{ \pm}$formed by connecting the endpoints of a path on $\gamma^{ \pm} \times S^{1}$ by an arc on $\hat{\Delta}^{ \pm} \backslash\left(\partial \gamma^{ \pm} \times \operatorname{int} D^{2}\right)$ so that $\beta^{ \pm}\left[a^{ \pm}\right]$links $G\left(S^{1} \times a^{ \pm}\right)$zero times; see Fig. B.3 Let $\hat{\beta}^{ \pm}$be a pushoff of $\beta^{ \pm}$along a normal vector field tangent to $\Delta^{ \pm}$. We see that $\beta^{ \pm}$and $\hat{\beta}^{ \pm}$bound embedded 2-disks $F^{ \pm}$and $\hat{F}^{ \pm}$in $D^{3}$, respectively, which are disjoint (that is to say, the aforementioned normal vector field extends to a normal vector field of $\left.F^{ \pm}\right)$. Then $\beta^{ \pm}\left[a^{ \pm}\right]$and $\hat{\beta}^{ \pm}\left[a^{ \pm}\right]$bound the disjoint, embedded 2-disks $F^{ \pm}\left[a^{ \pm}\right]$and $\hat{F}^{ \pm}\left[a^{ \pm}\right]$, respectively, which lie in $D^{3}\left[a^{ \pm}\right] \backslash G\left(S^{1} \times a^{ \pm}\right)$.

Since $f\left(S_{+}^{2}\right)$ is disjoint from $\Theta_{i}\left(N^{+}[-1,1]\right)$ and (we may assume) from a collar of the 2-disk $\Theta_{i}\left(F^{+}\left[a^{+}\right]\right)$, observe that $F^{+}$may be constructed from a normal pushoff $\hat{E}^{+}$of $E^{+}$in $D^{3}$ by attaching a collar so that the intersections between $\Theta_{i}\left(F^{+}\left[a^{+}\right]\right)$ and $f\left(S_{+}^{2}\right)$ occur entirely on $\Theta_{i}\left(\hat{E}^{+}\left[a^{+}\right]\right)$. Similarly, since $f\left(S_{+}^{2}\right)$ is disjoint from $\Theta_{i}\left(N^{+}[-1,1]\right)$ and $\Theta_{i}\left(N^{-}[-1,1]\right)$, and (we may assume) from a collar of the 2-disk $\Theta_{i}\left(F^{-}\left[a^{-}\right]\right)$, the 2-disk $F^{-}$may be chosen to contain a normal pushoff $\hat{V}$ of $V$ in $D^{3}$ and a normal pushoff $\check{E}^{+}$of $E^{+}$in $D^{3}$ so that the intersections between $\Theta_{i}\left(F^{-}\left[a^{+}\right]\right)$ and $f\left(S_{+}^{2}\right)$ occur entirely on $\Theta_{i}\left(\check{E}^{+}\left[a^{-}\right]\right)$and $\Theta_{i}\left(\hat{V}\left[a^{-}\right]\right)$. From these observations, by Proposition 2.2, we have (by an appropriate choice of whiskers and orientations in $X_{-}$)

$$
\lambda_{+}\left(\Theta_{i}\left(F^{+}\left[a^{+}\right]\right)\right)=\lambda_{+}\left(\Theta_{i}\left(\hat{E}^{+}\left[a^{+}\right]\right)\right)
$$

and

$$
\lambda_{+}\left(\Theta_{i}\left(F^{-}\left[a^{-}\right]\right)\right)=\lambda_{+}\left(\Theta_{i}\left(\check{E}^{+}\left[a^{-}\right]\right)\right)+\lambda_{+}\left(\Theta_{i}\left(\hat{V}\left[a^{-}\right]\right)\right)
$$

in $\mathbb{Z}\left[s, s^{-1}\right]$. But, as $\Theta_{i}\left(\hat{E}^{+}\left[a^{+}\right]\right)$and $\Theta_{i}\left(\check{E}^{+}\left[a^{-}\right]\right)$are each normal pushoffs in $X_{-}$ of $E_{i}^{+}:=\Theta_{i}\left(E^{+}[0]\right)$, the 2-disk bounded by $\alpha_{i}^{+}$, we deduce from Eq. (B.1) that

$$
\lambda_{+}\left(\Theta_{i}\left(F^{+}\left[a^{+}\right]\right)\right)=q_{i}^{(1)}(s)
$$




\section{A. Lightfoot}

for some integer Laurent polynomial $q_{i}^{(1)}(s) \in \mathbb{Z}\left[s, s^{-1}\right]$ such that

$$
q_{i}^{(1)}(1)=f\left(S_{+}^{2}\right) \cdot E_{i}^{+}=\operatorname{lk}\left(f\left(S_{+}^{2}\right), \alpha_{i}^{+}\right) .
$$

Moreover, if $\left|f\left(S_{+}^{2}\right) \cap E_{i}^{+}\right|=1$ for some $i$, then (as we are free to choose the orientation and whisker of $\left.\Theta_{i}\left(F^{+}\left[a^{+}\right]\right)\right)$we may take $q_{i}^{(1)}(s)=1$. Similarly, as $\Theta_{i}\left(\hat{V}\left[a^{-}\right]\right)$is a normal pushoff of $V_{i}$, from Eq. (B.2) we have (by an appropriate choice of orientations and whiskers in $X_{-}$)

$$
\lambda_{+}\left(\Theta_{i}\left(F^{-}\left[a^{-}\right]\right)\right)=q_{i}^{(1)}(s)+\lambda_{+}\left(V_{i}^{c}\right),
$$

where $V_{i}^{c} \subset X_{-}$is obtained from $V_{i}$ (which has boundary on $f\left(S_{-}^{2}\right)$ ) by removing a collar in $S^{4} \backslash f\left(S_{+}^{2}\right)$.

From the (embedded) punctured torus $\Delta^{ \pm}$, we remove the interior of the annulus bounded by $\beta^{ \pm} \cup \hat{\beta}^{ \pm}$and attach the 2-disks $F^{ \pm} \cup \hat{F}^{ \pm}$. We thus obtain an embedded 2-disk $\hat{A}^{ \pm} \subset D^{3}$ which has boundary $\hat{\delta}^{ \pm}$and is such that $\hat{A}^{ \pm}\left[a^{ \pm}\right]$ lies in $D^{3}\left[a^{ \pm}\right] \backslash G\left(S^{1} \times a^{ \pm}\right)$. Consequently, $\Theta_{i}\left(\hat{A}^{ \pm}\left[a^{ \pm}\right]\right)$is an embedded 2-disk in $X_{-}$with boundary $\Theta_{i}\left(\hat{\delta}^{ \pm}\left[a^{ \pm}\right]\right)$, obtained from the embedded punctured torus $\Theta_{i}\left(\Delta^{ \pm}\left[a^{ \pm}\right]\right) \subset X_{-} \backslash f\left(S_{+}^{2}\right)$ by applying the construction of Lemma 2.8 with the 2-disk $\Theta_{i}\left(F^{ \pm}\left[a^{ \pm}\right]\right)$and its normal pushoff $\Theta_{i}\left(\hat{F}^{ \pm}\left[a^{ \pm}\right]\right)$.

Now, for an interior point $z^{ \pm}$on $\gamma^{ \pm}$, the loop $z^{ \pm} \times S^{1} \subset \gamma^{ \pm} \times S^{1}$ is dual to $\beta^{ \pm}$and $\hat{\beta}^{ \pm}$on $\Delta^{ \pm}$, and is meridinal to $\hat{G}$ in $D^{3} \times D^{1}$. Thus, the loop $\Theta_{i}\left(z^{ \pm} \times S^{1}\right)$ is dual to $\partial \Theta_{i}\left(F^{ \pm}\left[a^{ \pm}\right]\right)$and $\partial \Theta_{i}\left(\hat{F}^{ \pm}\left[a^{ \pm}\right]\right)$on $\Theta_{i}\left(\Delta^{ \pm}\left[a^{ \pm}\right]\right)$, and represents $s$ or $s^{-1}$ in $\pi_{1}\left(X_{-}\right)=\mathbb{Z}\langle s\rangle$. By Lemma 2.8(i) and Eqs. B.3 B.5), then, we have (by an appropriate choice of orientations and whiskers in $X_{-}$)

$$
\lambda_{+}\left(\Theta_{i}\left(\hat{A}^{+}\left[a^{+}\right]\right)\right)=(1-s) q_{i}^{(1)}(s)
$$

and

$$
\lambda_{+}\left(\Theta_{i}\left(\hat{A}^{-}\left[a^{-}\right]\right)\right)=(1-s) q_{i}^{(1)}(s)+(1-s) \lambda_{+}\left(V_{i}^{c}\right),
$$

where $q_{i}^{(1)}(1)=\operatorname{lk}\left(f\left(S_{+}^{2}\right), \alpha_{i}^{+}\right)$.

We proceed to attach an annulus to each of the 2-disks $\hat{A}^{ \pm}\left[a^{ \pm}\right]$and $\hat{A}^{ \pm}\left[b^{ \pm}\right]$so to obtain 2-disks with which to surger the linking torus $T^{ \pm}$using the construction of Lemma 2.8.

In Fig. B.4, we have illustrated an oriented circle $\delta_{a}^{ \pm}$on $T^{ \pm} \subset N^{ \pm}[-1,1]$ which intersects $D^{3}[-1]$ and $D^{3}[1]$ each in an arc, and appears as a pair of points in $D^{3}[t]$ for $t \in(-1,1)$. We have also illustrated a normal pushoff $\delta_{b}^{ \pm}$of $\delta_{a}^{ \pm}$on $T^{ \pm}$. Let $\hat{T}^{ \pm}$ denote the annulus on $T^{ \pm}$bounded by $\delta_{a}^{ \pm} \cup \delta_{b}^{ \pm}$. Note that the pair $\delta_{a}^{ \pm} \cup \delta_{b}^{ \pm}$is isotopic in $N^{ \pm}[-1,1] \backslash \hat{G}$ to the link $\delta_{a}^{ \pm \prime} \cup \delta_{b}^{ \pm \prime}$ in $N^{ \pm}[-1,1] \backslash \hat{G}$ which we have also illustrated in Fig. [B.4 We then see that the annulus $\hat{T}^{ \pm}$is homotopic in $N^{ \pm}[-1,1] \backslash \hat{G}$ to the annulus $\hat{\delta}^{ \pm}\left[a^{ \pm}, b^{ \pm}\right]$by a homotopy of $S^{1} \times I$ whose restriction to $S^{1} \times\{0,1\}$ is a homotopy from $\delta_{a}^{ \pm} \cup \delta_{b}^{ \pm}$to the link $\hat{\delta}^{ \pm}\left[a^{ \pm}\right] \cup \hat{\delta}^{ \pm}\left[b^{ \pm}\right]$through a sequence of links, except for one singular link where the two components pass through each other. 

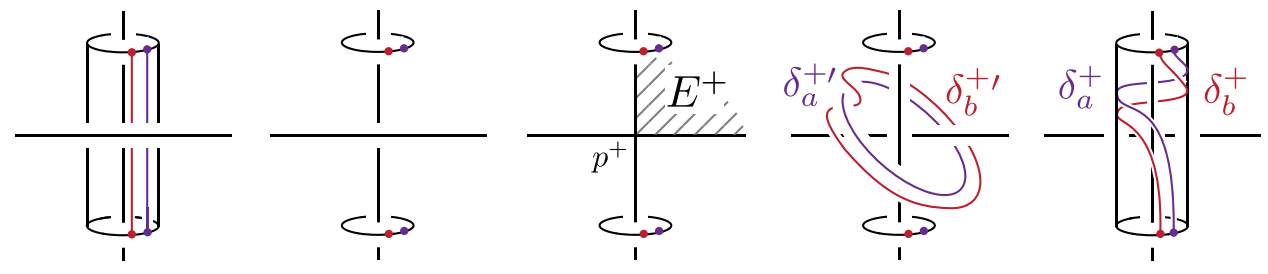

$x_{4}=-1$

$x_{4}=0$

$x_{4}=1$

(a) The torus $T^{+}$in $N^{+} \times D^{1}$

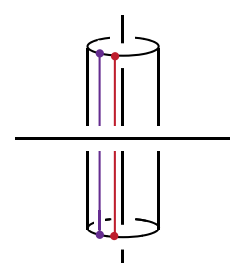

$x_{4}=-1$

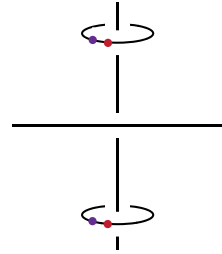

(b) The torus $T^{-}$in $N^{-} \times D^{1}$
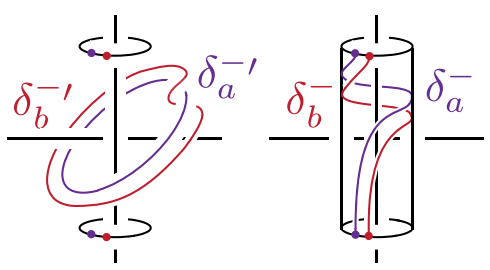

$x_{4}=1$

Fig. B.4.

That is, we can find a regular homotopy $K^{ \pm}:\left(S^{1} \times I\right) \times I \rightarrow N^{ \pm}[-1,1] \backslash \hat{G}$ such that

(1) $K_{0}^{ \pm}\left(S^{1} \times I\right)=\hat{T}^{ \pm}, K_{0}^{ \pm}\left(S^{1} \times 0\right)=\delta_{a}^{ \pm}, K_{0}^{ \pm}\left(S^{1} \times 1\right)=\delta_{b}^{ \pm}$;

(2) $K_{1}^{ \pm}\left(S^{1} \times I\right)=\hat{\delta}^{ \pm}\left[a^{ \pm}, b^{ \pm}\right], K_{1}^{ \pm}\left(S^{1} \times 0\right)=\hat{\delta}^{ \pm}\left[a^{ \pm}\right], K_{1}^{ \pm}\left(S^{1} \times 1\right)=\hat{\delta}^{ \pm}\left[b^{ \pm}\right]$; and

(3) $K_{t}^{ \pm}\left(S^{1} \times\{0,1\}\right)$ is a link for all $t \in I$ except at one value $t^{\prime}$, where $K_{t^{\prime}}^{ \pm}\left(S^{1} \times\right.$ $\{0,1\}$ ) has precisely with one transverse self-intersection point, arising as an intersection point between $K_{t^{\prime}}^{ \pm}\left(S^{1} \times 0\right)$ and $K_{t^{\prime}}^{ \pm}\left(S^{1} \times 1\right)$. Denote this intersection point by $w^{ \pm}$.

We may further suppose that the image of the homotopy $K^{ \pm}$lies in a tubular neighborhood $\left(\approx S^{1} \times D^{3}\right)$ of $\delta_{a}^{ \pm}$in $N^{ \pm}[-1,1] \backslash \hat{G}$. Hence, in particular, we may assume that for each $t \in(0,1)$ the image of $K_{t}^{ \pm}$is disjoint from each of $\hat{A}^{ \pm}\left[a^{ \pm}\right]$and $\hat{A}^{ \pm}\left[b^{ \pm}\right]$.

Attaching the annuli $K^{ \pm}\left(\left(S^{1} \times 0\right) \times I\right)$ and $K^{ \pm}\left(\left(S^{1} \times 1\right) \times I\right)$ to the 2-disks $\hat{A}^{ \pm}\left[a^{ \pm}\right]$and $\hat{A}^{ \pm}\left[b^{ \pm}\right]$along $\hat{\delta}^{ \pm}\left[a^{ \pm}\right]=K^{ \pm}\left(S^{1} \times 0 \times 1\right)$ and $\hat{\delta}^{ \pm}\left[b^{ \pm}\right]=K^{ \pm}\left(S^{1} \times 1 \times 1\right)$, respectively, we obtain embedded 2 -disks

$$
\Omega_{a}^{ \pm}=\hat{A}^{ \pm}\left[a^{ \pm}\right] \bigcup_{\hat{\delta}^{ \pm}\left[a^{ \pm}\right]} K^{ \pm}\left(\left(S^{1} \times 0\right) \times I\right)
$$

and

$$
\Omega_{b}^{ \pm}=\hat{A}^{ \pm}\left[b^{ \pm}\right] \bigcup_{\hat{\delta}^{ \pm}\left[b^{ \pm}\right]} K^{ \pm}\left(\left(S^{1} \times 1\right) \times I\right)
$$




\section{A. Lightfoot}

in $D^{4} \backslash \hat{G}$. Observe that their respective boundaries $\partial \Omega_{a}^{ \pm}=\delta_{a}^{ \pm}$and $\partial \Omega_{b}^{ \pm}=\delta_{b}^{ \pm}$lie on $T^{ \pm}$.

By construction, $\Omega_{a}^{ \pm}$and $\Omega_{b}^{ \pm}$intersect precisely once, transversely, at the intersection point $w^{ \pm}$between $K^{ \pm}\left(\left(S^{1} \times 0\right) \times I\right)$ and $K^{ \pm}\left(\left(S^{1} \times 1\right) \times I\right)$. Also, since the image of $K^{ \pm}$lies in $N^{ \pm}[-1,1]$, the intersections between $\Theta_{i}\left(\Omega_{a}^{ \pm}\right)$and $f\left(S_{+}^{2}\right)$ lie precisely on $\Theta_{i}\left(\hat{A}^{ \pm}\left[a^{ \pm}\right]\right)$. Consequently, by Proposition 2.2 we have (after an appropriate choice of orientations and whiskers in $X_{-}$)

$$
\lambda\left(\Theta_{i}\left(\Omega_{a}^{ \pm}\right)\right)=\lambda_{+}\left(\Theta_{i}\left(\hat{A}^{ \pm}\left[a^{ \pm}\right]\right)\right) .
$$

Now, let $A^{ \pm}$be the 2 -sphere in $D^{4} \backslash \hat{G}$ obtained by removing from the (embedded) torus $T^{ \pm}$the interior of the annulus $\hat{T}^{+}$(bounded by $\delta_{a}^{ \pm} \cup \delta_{b}^{ \pm}$) and attaching the embedded 2-disks $\Omega_{a}^{ \pm} \cup \Omega_{b}^{ \pm}$. Then $A^{ \pm}$is immersed and self-transverse in $D^{4}$, with a single double point: the point $w^{ \pm}$.

Wishing to apply Lemma 2.8, define a homotopy from $\Omega_{a}^{ \pm}$to $\Omega_{b}^{ \pm}$as follows. Let $c: S^{1} \times I \rightarrow D^{2}$ be a collar with $c\left(S^{1} \times 1\right)=\partial D^{2}$, and let $F_{\hat{A}^{ \pm}}: D^{2} \rightarrow D^{3}$ be an embedding with image $\hat{A}^{ \pm}$. Since $K^{ \pm}\left(S^{1} \times I \times I\right)$ and $\hat{A}^{ \pm}[a, b]$ each lie in $D^{4} \backslash \hat{G}$, from Eqs. B.8 (B.9), it is readily seen that

$$
\begin{aligned}
J_{t}^{ \pm}(c(x, s)) & =K^{ \pm}(x, t, s) & & \text { for }(x, s) \in S^{1} \times I, \\
J_{t}^{ \pm}(y) & =F_{\hat{A}^{ \pm}}(y)\left[a^{ \pm}+t\left(b^{ \pm}-a^{ \pm}\right)\right] & & \text {for } y \in D^{2},
\end{aligned}
$$

defines a homotopy $J^{ \pm}: D^{2} \times I \rightarrow D^{4} \backslash \hat{G}$ from $\Omega_{a}^{ \pm}$to $\Omega_{b}^{ \pm}$. Then

$$
A^{ \pm}=\left(T^{ \pm} \backslash \operatorname{int} \hat{T}^{ \pm}\right) \bigcup_{\delta_{a}^{ \pm} \cup \delta_{b}^{ \pm}} J^{ \pm}\left(D^{2} \times\{0,1\}\right) .
$$

Now, let $A_{i}^{ \pm}=\Theta_{i}\left(A^{ \pm}\right)$; then $A_{i}^{ \pm}$is an immersed, self-transverse 2-sphere in $D^{3}\left[t_{i}^{-}, t_{i}^{+}\right] \backslash f\left(S_{-}^{2}\right)$ constructed by surgering the embedded torus $T_{i}^{ \pm} \subset X_{-} \backslash f\left(S_{+}^{2}\right)$ using the 2-disks $\Theta_{i}\left(\Omega_{a}^{ \pm}\right) \cup \Theta_{i}\left(\Omega_{b}^{ \pm}\right) \subset X_{-}$. (In reference to the proof outline that followed the proposition statement, $\Theta_{i}\left(\Omega_{a}^{ \pm}\right)$is the desired 2-disk $O_{i}^{ \pm}$.) Furthermore, observe that a dual curve to $\delta_{a}^{ \pm}$and $\delta_{b}^{ \pm}$on the torus $T^{ \pm}$is meridinal to $\hat{G}$ in $D^{4}$, so a dual curve to $\Theta_{i}\left(\delta_{a}^{ \pm}\right)$and $\Theta_{i}\left(\delta_{a}^{ \pm}\right)$on the linking torus $\Theta_{i}\left(T^{ \pm}\right)$represents $s$ or $s^{-1}$ in $\pi_{1}\left(X_{-}\right)=\mathbb{Z}\langle s\rangle$. Thus, by Eq. B.11 and Lemma 2.8 (i), we have (after an appropriate choice of whiskers and orientations in $X_{-}$)

$$
\lambda_{+}\left(A_{i}^{+}\right)=(1-s) \lambda_{+}\left(\Theta_{i}\left(\Omega_{a}^{ \pm}\right)\right) .
$$

From Eqs. (B.6), (B.7) and (B.10), we therefore have

$$
\lambda_{+}\left(A_{i}^{+}\right)=(1-s)^{2} q_{i}(s)
$$

and

$$
\lambda_{+}\left(A_{i}^{-}\right)=(1-s)^{2} q_{i}(s)+(1-s)^{2} \lambda_{+}\left(V_{i}^{c}\right)
$$

for some $q_{i}(s) \in \mathbb{Z}\left[s, s^{-1}\right]$ such that $q_{i}(1)=\operatorname{lk}\left(f\left(S_{+}^{2}\right), \alpha_{i}^{+}\right)$. As observed earlier, if $\left|f\left(S_{+}^{2}\right) \cap E_{i}^{+}\right|=1$ for some $i$, then we may take $q_{i}(s)=1$. Moreover, since $\Theta_{i}\left(\Omega_{a}^{ \pm}\right)$ 
and $\Theta_{i}\left(\Omega_{b}^{ \pm}\right)$are embedded and intersect precisely once, we have from Lemma 2.8 (ii) that

$$
\mu\left(A_{i}^{ \pm}\right)=s \quad \bmod 2
$$

in $\mathbb{Z}_{2}[s]$. Hence $\lambda\left(A_{i}^{ \pm}, A_{i}^{ \pm}\right)=s+s^{-1} \bmod 2$.

Finally, by construction, $A_{i}^{ \pm}$is homologous to $T_{i}^{ \pm}$for each $i$, so the immersed 2-spheres $\left\{A_{i}^{+}, A_{i}^{-}\right\}_{i=1}^{d}$ generate $H_{2}\left(X_{-}\right)$and hence, by Lemma 3.3. represent a $\mathbb{Z}\left[s, s^{-1}\right]$-basis for $\pi_{2}\left(X_{-}\right)$.

\section{References}

[1] A. Bartels and P. Teichner, All two dimensional links are null homotopic, Geom. Topol. 3 (1999) 235-252.

[2] R. Fenn and D. Rolfsen, Spheres may link in 4-space, J. London Math. Soc. 34 (1986) 177-184.

[3] M. Freedman and R. Kirby, A geometric proof of Rochlin's theorem, Proc. Symp. Pure Math. AMS 32(2) (1978) 85-98.

[4] M. Freedman and F. Quinn, The Topology of 4-Manifolds, Princeton Mathematical Series, Vol. 39 (Princeton, NJ, 1990).

[5] N. Habegger and X.-S. Lin, The classification of links up to homotopy, J. Amer. Math. Soc. 3 (1990) 389-420.

[6] F. Hosokawa and A. Kawauchi, Proposals for unknotted surfaces in four-spaces, Osaka J. Math. 16 (1979) 233-248.

[7] S. Kamada, Vanishing of a certain kind of Vassiliev invariants of 2-knots, Proc. AMS 127(11) (1999) 3421-3426.

[8] P. Kirk, Link maps in the four sphere, in Proc. 1987 Siegen Topology Conf., SLNM 1350 (Springer, Berlin, 1988), 31-43.

[9] P. Kirk, Link homotopy with one codimension two component, Trans. Amer. Math. Soc. 319 (1990) 663-688.

[10] U. Koschorke, On link maps and their homotopy classification, Math. Ann. 286 (1990) 753-782.

[11] A. Kosinski, Differential Manifolds (Academic Press, San Diego, 1993).

[12] G.-S. Li, An invariant of link homotopy in dimension four, Topology 36 (1997) 881897.

[13] A. Lightfoot, On invariants of link maps in dimension four, J. Knot Theory Ramifications 251650060 (2016) [18 pages].

[14] W. S. Massey and D. Rolfsen, Homotopy classification of higher-dimensional links, Indiana Univ. Math. J. 34 (1985) 375-391.

[15] J. Milnor, Link groups, Ann. of Math 59 (1954) 177-195.

[16] A. Pilz, Verschlingungshomotopie von 2-Sphären im 4-dimensionalen Raum, Diploma thesis, University of Seigen (1997).

[17] R. Schneiderman and P. Teichner, Higher order intersection numbers of 2-spheres in 4-manifolds, Algebr. Geom. Topol. 1 (2001) 1-29.

[18] G. P. Scott, Homotopy links, Abh. Math. Sem. Univ. Hamburg 32 (1968) 186-190.

[19] C. T. C. Wall, Surgery on Compact Manifolds (Academic Press, New York, 1970). 